\title{
Research Article \\ Quad-Quaternion MUSIC for DOA Estimation Using Electromagnetic Vector Sensors
}

\author{
Xiaofeng Gong, Zhiwen Liu, and Yougen Xu \\ Department of Electronic Engineering, Beijing Institute of Technology, Beijing 100081, China
}

Correspondence should be addressed to Yougen Xu, yougenxu@bit.edu.cn

Received 24 April 2008; Revised 22 October 2008; Accepted 22 December 2008

Recommended by Jacques Verly

A new quad-quaternion model is herein established for an electromagnetic vector-sensor array, under which a multidimensional algebra-based direction-of-arrival (DOA) estimation algorithm, termed as quad-quaternion MUSIC (QQ-MUSIC), is proposed. This method provides DOA estimation (decoupled from polarization) by exploiting the orthogonality of the newly defined "quadquaternion" signal and noise subspaces. Due to the stronger constraints that quad-quaternion orthogonality imposes on quadquaternion vectors, QQ-MUSIC is shown to offer high robustness to model errors, and thus is very competent in practice. Simulation results have validated the proposed method.

Copyright (c) 2008 Xiaofeng Gong et al. This is an open access article distributed under the Creative Commons Attribution License, which permits unrestricted use, distribution, and reproduction in any medium, provided the original work is properly cited.

\section{INTRODUCTION}

A "complete" electromagnetic (EM) vector sensor comprises six collocated and orthogonally oriented EM sensors (e.g., short dipole and small loop), and provides complete electric and magnetic field measurements induced by an EM incidence [1-3]. An "incomplete" EM vector sensor with one or more components removed is also of high interest in some practical applications $[4,5]$. Numerous algorithms for direction-of-arrival (DOA) estimation using one or more EM vector sensors have been proposed. For example, vector sensor-based maximum likelihood strategy was addressed in [6-9], multiple signal classification (MUSIC [10]) was extended for both incomplete and complete EM vector-sensor arrays in [11-16], subspace fitting technique was reconsidered for incomplete EM vector sensors in $[17,18]$, and estimation of signal parameters via rotational invariance techniques (ESPRIT [19]) was revised for EM vector sensor(s) in [20-26]. The identifiability issue of EM vector sensor-based DOA estimation has been discussed in [27-29]. Some other related work can be found in [30-34]. In all the contributions mentioned above, complex-valued vectors are used to represent the output of each EM vector sensor in the array, and the collection of an EM vector-sensor array is arranged via concatenation of these vectors into a "long vector." Consequently, the corresponding algorithms somehow destroy the vector nature of incident signals carrying multidimensional information in space, time, and polarization.

More recently, a few efforts have been made on characterizing the output of vector sensors within a hypercomplex framework, wherein hypercomplex values, such as quaternions and biquaternions, are used to retain the vector nature of each vector sensor [35-37]. In particular, singular value decomposition technique was extended for quaternion matrices in [35] using three-component vector sensors. Quaternion-based MUSIC variant (Q-MUSIC) was proposed in [36] by using two-component vector sensors. Biquaternion-based MUSIC (BQ-MUSIC) was proposed in [37] by employing three-component vector sensors. The advantage of using quaternions and biquaternions for vector sensors is that the local vector nature of a vector-sensor array is preserved in multiple imaginary parts, and thus could result in a more compact formalism and a better estimation of signal subspace [36, 37]. More importantly, from the algebraic point of view, the algebras of quaternions and biquaternions are associative division algebras using specified norms [38], and therefore are convenient to use in the modeling and analysis of vector-sensor array processing. However, it is important to note that quaternions and biquaternions deal with only four-dimensional (4D) and 
(8D) algebras, respectively, while a full characterization of the sensor output for complete six-component EM vector sensors requires an algebra with dimensions equal to 12 or more.

Unfortunately, not all algebras having 12 or more dimensions are associative division algebras. For example, sedenions, as a well-known 16D algebra, are neither an associative algebra nor a division algebra [39], and thus are not suitable for the modeling and analysis of vector sensors. In this paper, we use a specific 16D algebraquad-quaternions algebra [40-42] to model the output of six-component EM vector sensor(s) [3]. This 16D quadquaternion algebra can be proved to be an associative division algebra, and thus is well adapted to the modeling and analysis of complete EM vector sensors. More precisely, We redefine the array manifold, signal subspace, and noise subspace from a quad-quaternion perspective, and propose a quad-quaternion-based MUSIC variant (QQMUSIC) for DOA estimation by recognizing and exploiting the quad-quaternion orthogonality between the quadquaternion signal and noise subspaces. QQ-MUSIC here is shown to be more attractive in the presence of two typical model errors, that is, sensor position error and sensor orientation error, which are often encountered in practice.

The rest of the paper is organized as follows. In Section 2, we present introductions on quad-quaternions and quadquaternion matrices. In Section 3, the quad-quaternionbased MUSIC algorithm is presented. In Section 4, we compare the proposed algorithm with some existing methods by simulations. Finally, we conclude the paper in Section 5.

Since this paper concerns several different hypercomplex values, we here summarize the symbols of values that will appear in subsequent sections in Table 1.

\section{QUAD-QUATERNIONS AND QUAD-QUATERNION MATRICES}

In this section, we introduce the algebra of quad-quaternions, and represent some results related to quad-quaternion matrices. The algebras of quaternions and biquaternions are introduced in detail in [35-38] and thus are not addressed here.

\subsection{Quad-quaternions and quad-quaternion matrices}

Quad-quaternion algebras are a class of 16D algebras [40], which were first considered by Albert since the 1930s [41]. (The quad-quaternion algebras mentioned in this paper are termed as the generalized biquaternion algebras in [40-42]). The quad-quaternion algebra is defined as follows.

Definition 1 (see [42]). Denote $\mathbb{H}_{\left(a_{n}, b_{n}\right)}$ the quaternion algebra over bases $\left\{1, i_{n}, j_{n}, k_{n}\right\}$, where $i_{n}^{2}=a_{n}, j_{n}^{2}=b_{n}$, $i_{n} j_{n}=-j_{n} i_{n}=k_{n}$, and $a_{n}, b_{n}$ are nonzero real numbers, $n=1,2$, then a quad-quaternion algebra over real numbers is the tensor product $[40]$ of $\mathbb{U}_{\left(a_{1}, b_{1}\right)}$ and $\mathbb{U}_{\left(a_{2}, b_{2}\right)}$, denoted by

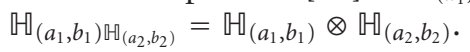

By definition, we can see that any element $p \in$ $\mathbb{H}_{\left(a_{1}, b_{1}\right) \mathbb{H}_{\left(a_{2}, b_{2}\right)}}$ can be expressed as

$$
\begin{aligned}
p= & \left(p_{00}+I p_{01}+J p_{02}+K p_{03}\right) \\
& +i\left(p_{10}+I p_{11}+J p_{12}+K p_{13}\right) \\
& +j\left(p_{20}+I p_{21}+J p_{22}+K p_{23}\right) \\
& +k\left(p_{30}+I p_{31}+J p_{32}+K p_{33}\right)
\end{aligned}
$$

where

$$
\begin{gathered}
i^{2}=a_{1}, \quad j^{2}=b_{1}, \quad k^{2}=-a_{1} b_{1}, \\
I^{2}=a_{2}, \quad J^{2}=b_{2}, \quad K^{2}=-a_{2} b_{2}, \\
i j=-j i=k, \quad I J=-J I=K, \\
k i=-i k=j \cdot\left(-a_{1}\right), \quad K I=-I K=J \cdot\left(-a_{2}\right), \\
j k=-k j=i \cdot\left(-b_{1}\right), \quad J K=-K J=I \cdot\left(-b_{2}\right), \\
l L=L l,
\end{gathered}
$$

where $l=i, j, k$ and $L=I, J, K$.

Denote the classical Hamilton quaternions by 메 [43], and consider the following particular case $a_{1}=b_{1}=a_{2}=$ $b_{2}=-1$, so that $\mathbb{H}_{\left(a_{1}, b_{1}\right)}=\mathbb{H}_{\left(a_{2}, b_{2}\right)}=\mathbb{H}$. Then, with an appropriate choice of norm, the tensor product of $\mathbb{M}$ and $\mathbb{M}$, denoted by $\mathbb{H}_{\mathbb{H}}=\mathbb{H} \otimes \mathbb{H}$, can be proved to be a division algebra according to [42, Theorem 4.3], so that zero divisors do not exist (note that the quad-quaternions herein mentioned are labeled as the generalized biquaternions in [42], which are different from the classical biquaternions). Since the algebra of quad-quaternions is always an associative algebra [41], then $\mathbb{U}_{\mathbb{H}}=\mathbb{U} \otimes \mathbb{U}$ is an associative division algebra.

Furthermore, from (1) we can see that $p \in \mathbb{H}_{\mathbb{H}}$ can be interpreted as a quaternion with quaternionic coefficients. In addition, if $p_{m n}=0$ for all $m, n=0,1,2,3, p$ is called a zero quad-quaternion, denoted by $p=0$. If $p_{00}=1$, and all the other coefficients are zero, then $p$ is called an identity quadquaternion, denoted by $p=1$. In addition, $p_{00}$ is called the scalar part of $p$, denoted by $S(p)$, while the vector part of $p$ is given by $V(p)=p-S(p)$.

Besides the expression in (1), a quad-quaternion $p \in \mathbb{M}_{\mathbb{H}}$ can as well be expressed as

$$
p=p_{0}+I p_{1}+J p_{2}+K p_{3}=b_{0}+I b_{1}
$$

where $p_{n}=p_{0 n}+i p_{1 n}+j p_{2 n}+k p_{3 n} \in \mathbb{M}, n=0,1,2,3 ; b_{m}=$ $p_{m}+J p_{m+2} \in \mathbb{H}_{\mathbb{C}^{(J)}}, m=0,1$. In particular, $p=b_{0}+I b_{1}$ can be considered as a quad-quaternion version of the CayleyDickson expression for quaternions and biquaternions in $[36,37]$. The definitions of addition and multiplication extend naturally from the case of biquaternion matrices, and thus are not addressed here.

From the geometric perspective, a quad-quaternion $p=$ $p_{0}+i p_{1}+j p_{2}+k p_{3}$ can be considered as a point in a $4 \mathrm{D}$ space spanned by $1, i, j, k$, as shown in Figure 1 . The difference from the quaternion case is that the $1, i, j, k$ coefficients of 
TABLE 1: Symbols of algebraic values.

\begin{tabular}{|c|c|}
\hline $\mathbb{R}$ & Real numbers \\
\hline $\mathbb{C}^{(L)}$ & Complex numbers with bases $\{1, L\}$, where $L=i, j, k, I, J, K$. In particular, $\mathbb{C}^{(i)}$ is denoted by $\mathbb{C}$. \\
\hline $\mathbb{H}_{(a, b)}$ & $\begin{array}{l}\text { Quaternions with bases }\{1, i, j, k\} \text { such that } i^{2}=a, j^{2}=b \text { and } i j=-j i=k \text {, where } a \text { and } b \text { are nonzero real numbers. In } \\
\text { particular, } \mathbb{H}=\mathbb{U}_{(-1,-1)} \text { corresponds to the classical Hamilton quaternions }\end{array}$ \\
\hline $\mathbb{\mathbb { H } ^ { ( 1 ) }}, \mathbb{\mathbb { W } ^ { ( 2 ) }}$ & $\begin{array}{l}\mathbb{U}^{(1)} \text { denotes Hamilton's quaternions with bases }\{1, i, j, k\} ; \mathbb{W}^{(2)} \text { denotes the Hamilton quaternions with bases }\{1, I, J, K\} \text {. } \\
\text { In particular, we denote } \mathbb{U}^{(1)} \text { by } \mathbb{U} \text {. }\end{array}$ \\
\hline $\mathbb{W}_{\mathbb{C}^{(L)}}$ & $\begin{array}{l}\text { Biquaternions with bases }\{1, i, j, k, L, L i, L j, L k\}, L=I, J, K \text {, such that } i^{2}=-1, j^{2}=-1, i j=-j i=k \text { and } l L=L l \text {, where } \\
l=i, j, k \text {. In particular, we denote } \mathbb{U}_{\mathbb{C}^{(I)}} \text { by } \mathbb{U}_{\mathbb{C}} \text {. }\end{array}$ \\
\hline $\mathbb{U}_{\mathbb{H}}$ & $\begin{array}{l}\text { Quad-quaternions with bases }\{1, i, j, k, I, I i, I j, I k, J, J i, J j, J k, K, K i, K j, K k\} \text { such that } i^{2}=j^{2}=I^{2}=J^{2}=-1 \text {, } \\
i j=-j i=k, I J=-J I=K \text {, and } l L=L l \text {, where } l=i, j, k \text { and } L=I, J, K \text {. }\end{array}$ \\
\hline
\end{tabular}

$p$ are not real values, but four individual quaternions which can be considered as four subpoints in a 4D hypospace spanned by $1, I, J, K$. Therefore, quite similar to quaternion rotations [38], we can interpret quad-quaternion multiplications as a more complex 16D "quad-quaternion rotations" which involve both $4 \mathrm{D}$ rotations(quaternion multiplications) and combination of 4D points (quaternion additions) in spaces spanned by $1, i, j, k$ and $1, I, J, K$.

Definition 2. A quad-quaternion matrix $\mathbf{Q} \in\left(\mathbb{H}_{\mathbb{Q}}\right)^{M \times N}$ is a matrix with $M$ rows and $N$ columns of which each element is a quad-quaternion $q_{m, n} \in \mathbb{H}_{\mathbb{H}}, m=1,2, \ldots, M, n=$ $1,2, \ldots, N$. In particular, an $N$ dimensional quad-quaternion column (row) vector can be considered as an $N \times 1(1 \times$ $N)$ quad-quaternion matrix. In this paper, quad-quaternion vectors are specifically referred to as column vectors. Similar to the case of quad-quaternion scalars, a quad-quaternion matrix $\mathbf{Q} \in\left(\mathbb{H}_{\mathbb{H}}\right)^{M \times N}$ can be expressed as follows:

$$
\begin{aligned}
\mathbf{Q}= & \left(\mathbf{Q}_{00}+I \mathbf{Q}_{01}+J \mathbf{Q}_{02}+K \mathbf{Q}_{03}\right) \\
& +i\left(\mathbf{Q}_{10}+I \mathbf{Q}_{11}+J \mathbf{Q}_{12}+K \mathbf{Q}_{13}\right) \\
& +j\left(\mathbf{Q}_{20}+I \mathbf{Q}_{21}+J \mathbf{Q}_{22}+K \mathbf{Q}_{23}\right) \\
& +k\left(\mathbf{Q}_{30}+I \mathbf{Q}_{31}+J \mathbf{Q}_{32}+K \mathbf{Q}_{33}\right) \\
= & \mathbf{Q}_{0}+I \mathbf{Q}_{1}+J \mathbf{Q}_{2}+K \mathbf{Q}_{3}=\mathbf{B}_{0}+I \mathbf{B}_{1},
\end{aligned}
$$

where $\mathbf{Q}_{n_{1} n_{2}} \in \mathbb{R}^{M \times N}, n_{1}, n_{2}=0,1,2,3, \mathbf{Q}_{n} \in \mathbb{H}^{M \times N}$, $n=0,1,2,3$, and $\mathbf{B}_{0}, \mathbf{B}_{1} \in\left(\mathbb{H}_{\mathbb{C}^{(J)}}\right)^{M \times N}$. Similar to quadquaternion scalars, the scalar and vector parts of $\mathbf{Q}$ are given by $S(\mathbf{Q})=\mathbf{Q}_{00}$ and $V(\mathbf{Q})=\mathbf{Q}-S(\mathbf{Q})$, respectively.

In the following discussion, we mainly focus on results related to quad-quaternion matrices and vectors. The results related to scalars can be directly obtained by considering a quad-quaternion scalar as a $1 \times 1$ quad-quaternion "matrix."

\subsection{Previous relevant results}

In this section, we present some results that are directly generalized from quaternion or biquaternion results. All the lemmas in this section can be proved similarly to their quaternion and biquaternion counterparts in [35-37], and thus are not included in this paper.

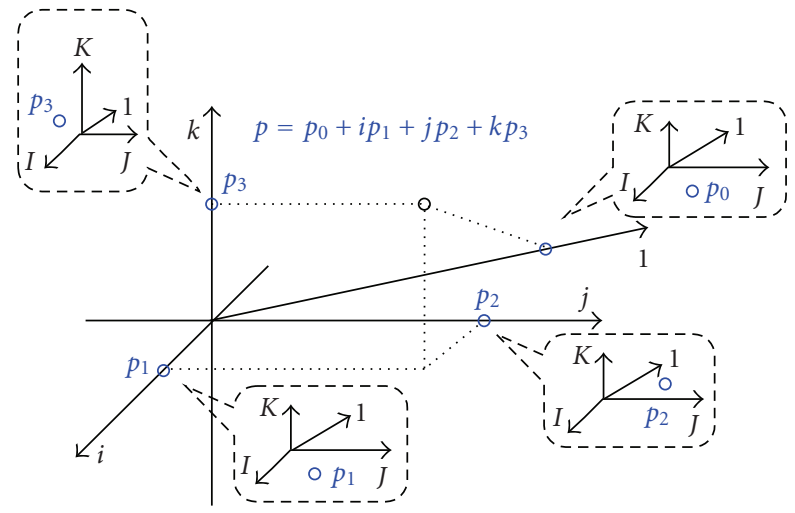

Figure 1: The geometric illustration of quad-quaternions.

Definition 3 ([37, Definition 2]). There exist four different conjugations for quad-quaternion matrices as follows.

(i) $\mathbb{C}$-conjugation $\mathbf{Q}^{\mathbb{C}}: \mathbf{Q}^{\mathbb{C}}=\mathbf{B}_{0}-I \mathbf{B}_{1}$;

(ii) $\mathbb{H}_{1}$-conjugation $\mathbf{Q}^{\star}: \mathbf{Q}^{\star}=\mathbf{Q}_{0}^{*}+I \mathbf{Q}_{1}^{*}+J \mathbf{Q}_{2}^{*}+K \mathbf{Q}_{3}^{*}$;

(iii) $\mathbb{H}_{2}$-conjugation $\mathbf{Q}^{*}: \mathbf{Q}^{*}=\mathbf{Q}_{0}-I \mathbf{Q}_{1}-J \mathbf{Q}_{2}-K \mathbf{Q}_{3}$;

(iv) Total-conjugation $\overline{\mathbf{Q}}: \overline{\mathbf{Q}}=\mathbf{Q}_{0}^{*}-I \mathbf{Q}_{1}^{*}-J \mathbf{Q}_{2}^{*}-K \mathbf{Q}_{3}^{*}$,

where $\mathbf{Q}_{n}^{*}$ denotes the quaternion conjugation of $\mathbf{Q}_{n}, n=$ $0,1,2,3$, as given in [36].

Definition 4 (from [37]). The transpose of $\mathbf{Q}=\mathbf{Q}_{0}+I \mathbf{Q}_{1}+$ $J \mathbf{Q}_{2}+K \mathbf{Q}_{3} \in\left(\mathbb{H}_{\mathbb{H}}\right)^{M \times N}$, denoted by $\mathbf{Q}^{T} \in\left(\mathbb{H}_{\mathbb{Q}}\right)^{N \times M}$, is defined as $\mathbf{Q}^{T} \triangleq \mathbf{Q}_{0}^{T}+I \mathbf{Q}_{1}^{T}+J \mathbf{Q}_{2}^{T}+K \mathbf{Q}_{3}^{T}$, where $\mathbf{Q}_{n}^{T}$ denotes the quaternion transpose of $\mathbf{Q}_{n}, n=0,1,2,3$. Then we have the following four different conjugated transposes.

(i) $\mathbb{C}$-conjugated transpose $\mathbf{Q}^{\triangleleft}$ : $\mathbf{Q}^{\triangleleft}=\left(\mathbf{Q}^{\mathbb{C}}\right)^{T}=\left(\mathbf{Q}^{T}\right)^{\mathbb{C}}$;

(ii) $\mathbb{M}_{1}$-conjugated transpose $\mathbf{Q}^{H_{1}}: \mathbf{Q}^{H_{1}}=\left(\mathbf{Q}^{\star}\right)^{T}=\left(\mathbf{Q}^{T}\right)^{\star}$;

(iii) $\mathbb{M}_{2}$-conjugated transpose $\mathbf{Q}^{H_{2}}: \mathbf{Q}^{H_{2}}=\left(\mathbf{Q}^{*}\right)^{T}=\left(\mathbf{Q}^{T}\right)^{*}$;

(iv) Total-conjugated transpose $\mathbf{Q}^{H}: \mathbf{Q}^{H}=(\overline{\mathbf{Q}})^{T}=\overline{\mathbf{Q}^{T}}$.

Definition 5 ([37, Definition 3]). The norm of a quadquaternion $p=p_{0}+I p_{1}+J p_{2}+K p_{3}$, denoted by $|p|$, is given by

$$
|p|=\sqrt{\left|p_{0}\right|^{2}+\left|p_{1}\right|^{2}+\left|p_{2}\right|^{2}+\left|p_{3}\right|^{2}}
$$


By definition, we can see that the following equation holds:

$$
\begin{aligned}
S(\bar{p} p)=S[ & \left.\left(p_{0}^{*}-I p_{1}^{*}-J p_{2}^{*}-K p_{3}^{*}\right)\left(p_{0}+I p_{1}+J p_{2}+K p_{3}\right)\right] \\
=S[ & \left(p_{0}^{*} p_{0}+p_{1}^{*} p_{1}+p_{2}^{*} p_{2}+p_{3}^{*} p_{3}\right) \\
& +I\left(p_{0}^{*} p_{1}+p_{3}^{*} p_{2}-p_{1}^{*} p_{0}-p_{2}^{*} p_{3}\right) \\
& +J\left(p_{0}^{*} p_{2}+p_{1}^{*} p_{3}-p_{2}^{*} p_{0}-p_{3}^{*} p_{1}\right) \\
& \left.+K\left(p_{0}^{*} p_{3}+p_{2}^{*} p_{1}-p_{3}^{*} p_{0}-p_{1}^{*} p_{2}\right)\right] \\
= & \left(p_{0}^{*} p_{0}+p_{1}^{*} p_{1}+p_{2}^{*} p_{2}+p_{3}^{*} p_{3}\right)=|p|^{2} .
\end{aligned}
$$

It is important to note that $|p q| \neq|p||q|$, so that quadquaternions do not form a normed algebra. We can further define the norm of a quad-quaternion vector $\mathbf{q} \in\left(\mathbb{H}_{\mathbb{Q}}\right)^{N \times 1}$ by

$$
\|\mathbf{q}\| \triangleq \sqrt{S\left(\mathbf{q}^{H} \mathbf{q}\right)} .
$$

Definition 6 (see (15) from [37]). Two quad-quaternion vectors $\mathbf{a}, \mathbf{b} \in\left(\mathbb{H}_{\mathbb{R}}\right)^{N \times 1}$ are said to be orthogonal if

$$
\mathbf{a}^{H} \mathbf{b}=0 \text {. }
$$

Definition 7 (see (17) from [37]). The adjoint matrix $\left(\chi_{Q} \in\right.$ $\left.\left(\mathbb{H}_{\mathbb{C}^{(J)}}\right)^{2 M \times 2 N}\right)$ of a quad-quaternion matrix $\mathbf{Q} \in\left(\mathbb{H}_{\mathbb{H}}\right)^{M \times N}=$ $\mathbf{B}_{0}+I \mathbf{B}_{1}\left(\right.$ where $\mathbf{B}_{0}, \mathbf{B}_{1} \in\left(\mathbb{H}_{\mathbb{C}^{(J)}}\right)^{M \times N}$ ) is given by

$$
\chi_{Q} \triangleq\left(\begin{array}{cc}
\mathbf{B}_{0} & \mathbf{B}_{1}^{*} \\
-\mathbf{B}_{1} & \mathbf{B}_{0}^{*}
\end{array}\right) .
$$

Let further $\Psi_{M} \triangleq\left[\mathbf{I}_{M},-I \cdot \mathbf{I}_{M}\right] \in\left(\mathbb{C}^{(I)}\right)^{M \times 2 M}$, where $\mathbf{I}_{M}$ is the identity matrix of size $M \times M$, then

$$
\mathbf{Q}=\frac{1}{2} \boldsymbol{\Psi}_{M} \boldsymbol{\chi}_{\mathrm{Q}} \boldsymbol{\Psi}_{N}^{H}
$$

where

$$
\begin{gathered}
\boldsymbol{\Psi}_{M} \boldsymbol{\Psi}_{M}^{H}=2 \mathbf{I}_{M}, \\
\chi_{Q} \boldsymbol{\Psi}_{N}^{H} \boldsymbol{\Psi}_{N}=\boldsymbol{\Psi}_{M}^{H} \boldsymbol{\Psi}_{M} \boldsymbol{\chi}_{Q} .
\end{gathered}
$$

Lemma 1 (from [35]). Consider two quad-quaternion matrices $\mathbf{A} \in\left(\mathbb{H}_{\mathbb{H}}\right)^{M \times N}$ and $\mathbf{B} \in\left(\mathbb{H}_{\mathbb{H}}\right)^{N \times L}$, and denote the adjoint matrices of $\mathrm{A}, \mathrm{B}$, and $\mathrm{AB}$ by $\chi_{A}, \chi_{B}$, and $\chi_{A B}$, respectively, then

$$
\chi_{A B}=\chi_{A} \cdot \chi_{B}
$$

Lemma 2 ([37, Lemma 1]). If $\mathbf{P}^{H}=\mathbf{P}$, then $\mathbf{P} \in\left(\mathbb{H}_{\mathbb{B}}\right)^{N \times N}$ is Hermitian. Then we note that the adjoint matrix of a Hermitian quad-quaternion matrix is also Hermitian.

Definition 8 (from [37]). If $\mathbf{Q u}=\mathbf{u} \lambda$, where $\mathbf{u} \in\left(\mathbb{H}_{\mathbb{q}}\right)^{N \times 1}$, $\lambda \in \mathbb{C}$, and $\mathbf{Q} \in\left(\mathbb{H}_{\mathbb{H}}\right)^{N \times N}$, then $\lambda$ and $\mathbf{u}$ are, respectively, the right eigenvalue and the associated right eigenvector of $\mathbf{Q}$.

Lemma 3 ([37, Lemma 2]). Denote the adjoint matrix of $\mathbf{Q} \in$ $\left(\mathbb{H}_{\mathbb{H}}\right)^{N \times N}$ by $\chi_{Q}$, if $\lambda \in \mathbb{C}$ and $\mathbf{u}_{b} \in\left(\mathbb{H}_{\mathbb{C}^{(J)}}\right)^{2 N \times 1}$ are the right eigenvalue and the associated right eigenvector of $\chi_{Q}$, then $\lambda$ and $\mathbf{u}=\Psi_{N} \mathbf{u}_{b}$ are the right eigenvalue and the associated right eigenvector of $\mathbf{Q}$.
Corollary 1 (from [37]). The eigenvalues of a Hermitian quad-quaternion matrix are real values. Consider a Hermitian quad-quaternion matrix $\mathbf{Q} \in\left(\mathbb{H}_{\mathbb{Q}}\right)^{N \times N}$ whose adjoint matrix $\chi_{Q}$ can be eigendecomposed as $\chi_{Q}=\mathbf{U}_{b} \mathbf{D} \mathbf{U}_{b}^{H}$, where $\mathbf{U}_{b} \in$ $\left(\mathbb{H}_{\mathbb{C}}\right)^{2 N \times 4 N}$, and $\mathbf{D} \in \mathbb{R}^{(4 N \times 4 N)}$ is a real diagonal matrix. The eigendecomposition of $\mathbf{Q}$ is then given by

$$
\mathbf{Q}=\mathbf{U D U}^{H}=\sum_{n=1}^{4 N} \lambda_{n} \mathbf{u}_{n} \mathbf{u}_{n}^{H},
$$

where $\mathbf{U}=(1 / \sqrt{2}) \Psi_{N} \mathbf{U}_{b} \in\left(\mathbb{M}_{\mathbb{H}}\right)^{N \times 4 N}, \lambda_{n}$ is the $n$th element of the diagonal of $\mathbf{D}, \mathbf{u}_{n}$ is the nth column vector of $\mathbf{U}$.

Lemma 4. The eigenvectors corresponding to different eigenvalues of a Hermitian quad-quaternion matrix are orthogonal.

\subsection{New definitions and lemmas for quad-quaternioins}

In this section, we introduce some new results related to quad-quaternions. For an easier reading of this section, all the results are given directly, while some of their proofs are summarized in the appendix for the reference of interested readers.

Definition 9. Let $\Lambda=\{1, i, j, k, I, I i, I j, I k, J, J i, J j, J k, K, K i$, $K j, K k\}$ and $\Gamma \subseteq \Lambda$, then the $\Gamma$-match of a quad-quaternion matrix $\mathbf{Q} \in\left(\mathbb{H}_{\mathbb{Q}}\right)^{M \times N}$, denoted by $S(\mathbf{Q} \mid \Gamma)$, is obtained by keeping the coefficients of the units in $\Gamma$ unchanged, and setting all the other coefficients to zero. The $\Gamma$-complement of $\mathbf{Q}$ is defined as $V(\mathbf{Q} \mid \Gamma) \triangleq \mathbf{Q}-S(\mathbf{Q} \mid \Gamma)$.

By definition, we know that the match and complement operations are used to select some desired parts of quadquaternions. For example, if $\Gamma=\{1, i, K\}$, then $S(\mathbf{Q} \mid \Gamma)=$ $\mathbf{Q}_{00}+i \mathbf{Q}_{10}+K \mathbf{Q}_{03}$, where $\mathbf{Q}_{00}, \mathbf{Q}_{10}, \mathbf{Q}_{03}$ are given in (4). Also it can be proved that $V\left(\mathbf{Q} \mid \Gamma^{\perp}\right)=S(\mathbf{Q} \mid \Gamma)$, where $\Gamma^{\perp}$ denotes the complement of $\Gamma$. Particularly, if $\Gamma=\{1\}, S(\mathbf{Q} \mid \Gamma)$ and $V(\mathbf{Q} \mid \Gamma)$ are equal to the scalar part and the vector part of $\mathrm{Q}$, respectively.

Definition 10. Let $\Lambda=\{1, i, j, k, I, I i, I j, I k, J, J i, J j, J k, K, K i$, $K j, K k\}$, and $\Gamma \subseteq \Lambda$, then the $\Gamma$-conjugation of a quadquaternion matrix $\mathbf{Q}=S(\mathbf{Q} \mid \Gamma)+V(\mathbf{Q} \mid \Gamma) \in\left(\mathbb{H}_{\mathbb{H}}\right)^{M \times N}$ is denoted by conj $(\mathbf{Q} \mid \Gamma)$, and defined as

$$
\operatorname{conj}(\mathbf{Q} \mid \Gamma) \triangleq S(\mathbf{Q} \mid \Gamma)-V(\mathbf{Q} \mid \Gamma) .
$$

It should be noted that the four conjugations given in Definition 3 are actually four special examples of the $\Gamma$ conjugation corresponding to different selections of $\Gamma$. For example, the $\mathbb{H}_{1}$-conjugation corresponds to $\Gamma=$ $\{1, I, J, K\}$, whereas the total-conjugation corresponds to $\Gamma=$ $\{i, j, k, I, J, K\}^{\perp}$.

Lemma 5. Given two sets $\Gamma_{1}, \Gamma_{2} \subseteq \Lambda$, we have

$$
\begin{aligned}
& \operatorname{conj}\left(\left(\operatorname{conj}\left(\mathbf{Q} \mid \Gamma_{1}\right)\right) \mid \Gamma_{2}\right) \\
& \quad=\operatorname{conj}\left(\mathbf{Q} \mid\left(\Gamma_{1} \cap \Gamma_{2}\right) \cup\left(\Gamma_{1} \cup \Gamma_{2}\right)^{\perp}\right),
\end{aligned}
$$


where $\Gamma_{1} \cap \Gamma_{2}$ and $\Gamma_{1} \cup \Gamma_{2}$ denote the intersection and union of $\Gamma_{1}$ and $\Gamma_{2}$, respectively.

Definition 11. Let $\Lambda=\{1, i, j, k, I, I i, I j, I k, J, J i, J j, J k, K, K i$, $K j, K k\}$ and $\Gamma \subseteq \Lambda$, the $\Gamma$-conjugated transpose of $\mathbf{Q}$ is given by $\operatorname{conj}(\mathbf{Q} \mid \Gamma)^{T}$. It is easy to prove that $\operatorname{conj}(\mathbf{Q} \mid \Gamma)^{T}=$ $\operatorname{conj}\left(\mathbf{Q}^{T} \mid \Gamma\right)$. Also, when $\Gamma=\Lambda, \mathbf{Q}^{T}=\operatorname{conj}(\mathbf{Q} \mid \Gamma)^{T}$. Similar to the $\Gamma$-conjugation, the four different conjugated transposes given in Definition 4 are four different examples corresponding to four different selections of $\Gamma$.

Definition 12. Quad-quaternion vectors $\mathbf{v}_{1}, \mathbf{v}_{2}, \ldots, \mathbf{v}_{N} \in$ $\left(\mathbb{H}_{\mathbb{H}}\right)^{M \times 1}$ are said to be right (left) linear dependent if there are scalars $\mu_{1}, \mu_{2}, \ldots, \mu_{N} \in \mathbb{H}_{\mathbb{q}}$ not all zero, such that $\mathbf{v}_{1} \mu_{1}+$ $\mathbf{v}_{2} \mu_{2}+\cdots+\mathbf{v}_{N} \mu_{N}=\mathbf{o}_{M \times 1}\left(\mu_{1} \mathbf{v}_{1}+\mu_{2} \mathbf{v}_{2}+\cdots+\mu_{N} \mathbf{v}_{N}=\right.$ $\left.\mathbf{o}_{M \times 1}\right)$. Moreover, if $\mathbf{v}_{1} \mu_{1}+\mathbf{v}_{2} \mu_{2}+\cdots+\mathbf{v}_{N} \mu_{N}=\mathbf{o}_{M \times 1}$ $\left(\mu_{1} \mathbf{v}_{1}+\mu_{2} \mathbf{v}_{2}+\cdots+\mu_{N} \mathbf{v}_{N}=\mathbf{o}_{M \times 1}\right)$ is true if and only if $\mu_{1}, \mu_{2}, \ldots, \mu_{N}$ are all zero, vectors $\mathbf{v}_{1}, \mathbf{v}_{2}, \ldots, \mathbf{v}_{N}$ are said to be right (left) linearly independent. Here, $\mathbf{o}_{N \times 1}$ is an $N \times 1$ zero vector. Obviously, since $\mathbf{v}_{1} \mu_{1} \neq \mu_{1} \mathbf{v}_{1}$ in most cases, the concept of right linear dependent (independent) is different from that of left linear dependent (independent).

Definition 13. Given a set of quad-quaternion vectors $\mathbf{v}_{1}, \mathbf{v}_{2}, \ldots, \mathbf{v}_{N} \in\left(\mathbb{H}_{\mathbb{H}}\right)^{M \times 1}$, if $\mathbf{v}_{1}, \mathbf{v}_{2}, \ldots, \mathbf{v}_{R}(R<N)$, are right (left) linearly independent and there exists an arbitrary vector $\mathbf{v}_{R+1} \in\left(\mathbb{H}_{\mathbb{H}}\right)^{M \times 1}$ such that $\mathbf{v}_{1}, \mathbf{v}_{2}, \ldots, \mathbf{v}_{R+1}$ are right (left) linearly dependent, then $\mathbf{v}_{1}, \mathbf{v}_{2}, \ldots, \mathbf{v}_{R}$ form a maximal right (left) linearly independent set. Furthermore, we define the right (left) rank of $\left\{\mathbf{v}_{1}, \mathbf{v}_{2}, \ldots, \mathbf{v}_{N}\right\}$ as $\operatorname{rank}_{R}\left(\left\{\mathbf{v}_{1}, \mathbf{v}_{2}, \ldots, \mathbf{v}_{R}\right\}\right) \triangleq R\left(\operatorname{rank}_{L}\left(\left\{\mathbf{v}_{1}, \mathbf{v}_{2}, \ldots, \mathbf{v}_{R}\right\}\right) \triangleq\right.$ $R)$.

Definition 14. Given a quad-quaternion matrix $\mathbf{P}=\left[\mathbf{p}_{1}\right.$, $\left.\mathbf{p}_{2}, \ldots, \mathbf{p}_{N}\right]$, where $\mathbf{p}_{n}$ is the $n$th column of $\mathbf{P}, n=$ $1,2, \ldots, N$. Then the right (left) rank of $\mathbf{P}$ is defined as $\operatorname{rank}_{R}(\mathbf{P}) \triangleq \operatorname{rank}_{R}\left(\left\{\mathbf{p}_{1}, \mathbf{p}_{2}, \ldots, \mathbf{p}_{N}\right\}\right)\left(\operatorname{rank}_{L}(\mathbf{P}) \triangleq\right.$ $\left.\operatorname{rank}_{L}\left(\left\{\mathbf{p}_{1}, \mathbf{p}_{2}, \ldots, \mathbf{p}_{N}\right\}\right)\right)$. In addition, we have the following lemma.

Lemma 6. Denote the adjoint matrix of $\mathbf{P} \in\left(\mathbb{H}_{\mathbb{Q}}\right)^{N \times N}$ by $\chi_{P}$, then

$$
\operatorname{rank}_{R}(\mathbf{P})=\frac{1}{2} \operatorname{rank}_{R}\left(\chi_{P}\right)\left(\operatorname{rank}_{L}(\mathbf{P})=\frac{1}{2} \operatorname{rank}_{L}\left(\chi_{P}\right)\right) .
$$

In the following discussion, we only consider the right rank, and we denote $\operatorname{rank}(\mathbf{P})=\operatorname{rank}_{R}(\mathbf{P})$.

Lemma 7. Denote the eigenvalue decomposition of a Hermitian quad-quaternion matrix $\mathbf{Q}$ by $\mathbf{Q}=\mathbf{U} \mathbf{U U}^{H}$, then we have

$$
\operatorname{rank}(\mathbf{Q})=\frac{1}{4} \operatorname{rank}(\mathbf{D})
$$

Definition 15. Given a set of orthogonal quad-quaternion vectors $\mathbf{v}_{1}, \mathbf{v}_{2}, \ldots, \mathbf{v}_{N}$, we can define the vector space $\mathfrak{R}$ spanned by $\mathbf{v}_{1}, \mathbf{v}_{2}, \ldots, \mathbf{v}_{N}$ as $\mathfrak{R} \triangleq\left\{\mathbf{v} \mid \mathbf{v}=\mathbf{v}_{1} \mu_{1}+\right.$ $\left.\mathbf{v}_{2} \mu_{2}+\cdots+\mathbf{v}_{N} \mu_{N}\right\}$, where $\mu_{1}, \mu_{2}, \ldots, \mu_{N}$ are arbitrary quad-quaternion scalars. $\mathfrak{R}$ can also be denoted as $\mathfrak{R}=$ $\operatorname{span}\left(\mathbf{v}_{1}, \mathbf{v}_{2}, \ldots, \mathbf{v}_{N}\right)$.

Lemma 8. If $\mathbf{v}_{1}, \mathbf{v}_{2}, \ldots, \mathbf{v}_{N}$ are $N$ eigenvectors of a Hermitian quad-quaternion matrix, then $\mathbf{v}_{1} \mu_{1}, \mathbf{v}_{2} \mu_{2}, \ldots, \mathbf{v}_{N} \mu_{N}$ are also a set of eigenvectors of this Hermitian quad-quaternion matrix, where $\mu_{1}, \mu_{2}, \ldots, \mu_{N}$ are nonzero quad-quaternions. Then, we have $\operatorname{span}\left(\mathbf{v}_{1}, \mathbf{v}_{2}, \ldots, \mathbf{v}_{N}\right)=\operatorname{span}\left(\mathbf{v}_{1} \mu_{1}, \mathbf{v}_{2} \mu_{2}, \ldots\right.$, $\left.\mathbf{v}_{N} \mu_{N}\right)$.

This lemma indicates that the indetermination of eigenvectors of a Hermitian quad-quaternion matrix does not impact their span. From the geometric perspective, when the eigenvector multiplies a nonzero scalar from the right side, all the elements of this eigenvector are rotated in the $16 \mathrm{D}$ quad-quaternion space (as shown in Figure 1) with the same quad-quaternion manner, and the proportional relationship between different elements does not change. Therefore, the intrinsic "structure" of this eigenvector is independent of the above-mentioned eigenvector indetermination.

\section{QUAD-QUATERNION MUSIC}

\subsection{Quad-quaternion model for EM vector sensors}

Let $(\theta, \varphi)$ and $(\gamma, \eta)$ be the azimuth-elevation 2D DOA (see Figure 2) and polarization of an EM signal, respectively, where $0<\theta \leq 2 \pi, 0 \leq \varphi \leq \pi$, and $0 \leq \gamma \leq \pi / 2,-\pi \leq \eta \leq \pi$. The output of an EM vector sensor then can be capsulated into the following quad-quaternion scalar:

$$
\begin{aligned}
p^{(\theta, \varphi, \gamma, \eta)}= & i\left[E_{x}^{(\theta, \varphi, \gamma, \eta)}+I H_{x}^{(\theta, \varphi, \gamma, \eta)}\right]+j\left[E_{y}^{(\theta, \varphi, \gamma, \eta)}+I H_{y}^{(\theta, \varphi, \gamma, \eta)}\right] \\
& +k\left[E_{z}^{(\theta, \varphi, \gamma, \eta)}+I H_{z}^{(\theta, \varphi, \gamma, \eta)}\right]
\end{aligned}
$$

where $E_{x}^{(\theta, \varphi, \gamma, \eta)}, E_{y}^{(\theta, \varphi, \gamma, \eta)}, E_{z}^{(\theta, \varphi, \gamma, \eta)} \in \mathbb{C}^{(J)}$, and $H_{x}^{(\theta, \varphi, \gamma, \eta)}$, $H_{y}^{(\theta, \varphi, \gamma, \eta)}, H_{z}^{(\theta, \varphi, \gamma, \eta)} \in \mathbb{C}^{(J)}$ are the three components of the electric vector and the magnetic vector, respectively, which are defined as [2]

$$
\left(\begin{array}{l}
E_{x}^{(\theta, \varphi, \gamma, \eta)} \\
E_{y}^{(\theta, \varphi, \gamma, \eta)} \\
E_{z}^{(\theta, \varphi, \gamma, \eta)} \\
H_{x}^{(\theta, \varphi, \gamma, \eta)} \\
H_{y}^{(\theta, \varphi, \gamma, \eta)} \\
H_{z}^{(\theta, \varphi, \gamma, \eta)}
\end{array}\right) \triangleq\left(\begin{array}{cc}
-\sin \theta & \cos \varphi \cos \theta \\
\cos \theta & \cos \varphi \sin \theta \\
0 & -\sin \varphi \\
-\cos \varphi \cos \theta & -\sin \theta \\
-\cos \varphi \sin \theta & \cos \theta \\
\sin \varphi & 0
\end{array}\right) \cdot \underbrace{\left(\begin{array}{c}
\cos \gamma \\
\sin \gamma e^{J \eta}
\end{array}\right)}_{\mathbf{h}_{\gamma, \eta} \in\left(\mathbb{C}^{(J)}\right)^{2 \times 1}} .
$$

Thus, (18) can be rewritten as 


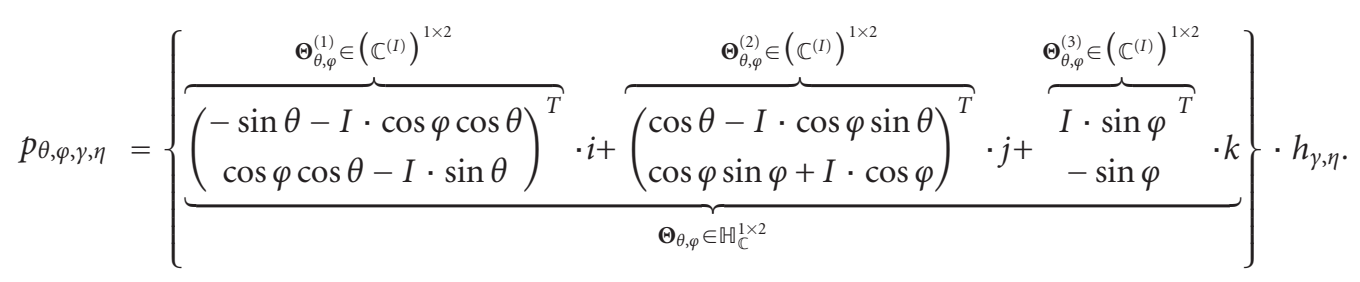

For an array of $N$ EM vector sensors, the spatial steering vector $\mathbf{d}_{\theta, \varphi}$ is given by

$$
\mathbf{d}_{\theta, \varphi}=\left[e^{J \cdot 2 \pi\left(\mathbf{k}_{1}^{T} \mathbf{e}_{\theta, \varphi} / \lambda\right)}, \ldots, e^{J \cdot 2 \pi\left(\mathbf{k}_{N}^{T} e_{\theta, \varphi} / \lambda\right)}\right]^{T},
$$

where $\mathbf{k}_{n}$ is the position vector of the $n$th EM vector sensor, $\mathbf{e}_{\theta, \varphi}$ is the propagation vector corresponding to $(\theta, \varphi), \lambda$ is the wavelength of incident signals. The steering vector of such an $\mathrm{N}$-element EM vector-sensor array then can be expressed as

$$
\begin{aligned}
\mathbf{a}_{\theta, \varphi, \gamma, \eta}= & p_{\theta, \varphi, \gamma, \eta} \mathbf{d}_{\theta, \varphi}=\left(\boldsymbol{\Theta}_{\theta, \varphi} \otimes \mathbf{d}_{\theta, \varphi}\right) \mathbf{h}_{\gamma, \eta} \\
= & \underbrace{\left(\Theta_{\theta, \varphi}^{(1)} \otimes \mathbf{d}_{\theta, \varphi}\right) \mathbf{h}_{\gamma, \eta}}_{\mathbf{a}_{\theta, \varphi, \gamma, \eta}^{(1)}} \cdot i+\underbrace{\left(\Theta_{\theta, \varphi}^{(2)} \otimes \mathbf{d}_{\theta, \varphi}\right) \mathbf{h}_{\gamma, \eta}}_{\mathbf{a}_{\theta, \varphi, \vartheta, \eta}^{(2)}} \cdot j \\
& +\underbrace{\left(\boldsymbol{\Theta}_{\theta, \varphi}^{(3)} \otimes \mathbf{d}_{\theta, \varphi}\right) \mathbf{h}_{\gamma, \eta}}_{\mathbf{a}_{\theta, \varphi, \gamma, \eta}^{(3)}} \cdot k,
\end{aligned}
$$

where " $\otimes$ " denotes the Kronecker product.

In the presence of $M$ narrowband, far-field, and completely polarized signals, the quad-quaternion model of an $\mathrm{N}$-element EM vector-sensor array has the following form:

$$
\begin{aligned}
\mathbf{x}(t) & =\sum_{m=1}^{M} \mathbf{a}_{m} s_{m}(t)+\mathbf{n}(t) \\
& =\sum_{m=1}^{M}\left[\left(\boldsymbol{\Theta}_{m} \otimes \mathbf{d}_{m}\right) \cdot \mathbf{h}_{m}\right] \cdot s_{m}(t)+\mathbf{n}(t),
\end{aligned}
$$

where $s_{m}(t) \in \mathbb{C}^{(J)}$ is the complex envelop of the $m$ th signal, and $\mathbf{n}(t)$ is the additive noise term, and $\mathbf{d}_{m}=\mathbf{d}_{\theta_{m}, \varphi_{m}}, p_{m}=$ $p_{\theta_{m}, \varphi_{m}, \gamma_{m}, \eta_{m}}, \boldsymbol{\Theta}_{m}=\boldsymbol{\Theta}_{\theta_{m}, \varphi_{m}}, \mathbf{h}_{m}=\mathbf{h}_{\gamma_{m}, \eta_{m}}$. It is assumed here that (1) all the incident signals are uncorrelated; (2) the noise is spatially white and uncorrelated with the signals; (3) steering vectors corresponding to different selections of $(\theta, \varphi, \gamma, \eta)$ are right linearly independent.

\subsection{Algorithm details}

We first define the quad-quaternion array manifold $\boldsymbol{\Phi}$ as the continuum of steering vector $\mathbf{a}_{\theta, \varphi, \gamma, \eta}$ in the angular parameter space of interest $\mathfrak{I}_{1}$ and the polarization parameter space of interest $\mathfrak{I}_{2}$. That is,

$$
\Phi \triangleq\left\{\mathbf{a}_{\theta, \varphi, \gamma, \eta},(\theta, \varphi) \in \mathfrak{I}_{1},(\gamma, \eta) \in \mathfrak{I}_{2}\right\} .
$$

Moreover, the signal subspace and noise subspace in quadquaternion case are defined as follows:

$$
\begin{gathered}
\mathfrak{R}_{s}=\operatorname{span}\left(\mathbf{a}_{1}, \ldots, \mathbf{a}_{M}\right), \\
\mathfrak{R}_{n}=\mathfrak{R}_{s}^{\perp} .
\end{gathered}
$$

Define the covariance matrix with quad-quaternion entries as

$$
\mathbf{R}_{x}=E\left[\mathbf{x}\left(t_{l}\right) \mathbf{x}^{H}\left(t_{l}\right)\right],
$$

where " $E$ " denotes expectation. From (23),

$$
\mathbf{R}_{x}=\sum_{m=1}^{M} \sigma_{m}^{2} \mathbf{a}_{m} \mathbf{a}_{m}^{H}+\mathbf{R}_{n}
$$

where $\sigma_{m}^{2}=E\left[s_{m}(t) s_{m}^{*}(t)\right]$ and $\mathbf{R}_{n}=E\left[\mathbf{n}(t) \mathbf{n}^{H}(t)\right]=\sigma_{n}^{2} \mathbf{I}_{N}$.

It can be easily proven according to Definition 14 that the rank of $\mathbf{A}=\left[\mathbf{a}_{1}, \mathbf{a}_{2}, \ldots, \mathbf{a}_{M}\right]$ is $M$, then in the absence of noise, the rank of $\mathbf{R}_{x}=\operatorname{diag}\left(\left[\sigma_{1}^{2}, \sigma_{2}^{2}, \ldots, \sigma_{M}^{2}\right]\right) \mathbf{A} \mathbf{A}^{H}$ is $M$, and the column vectors of $\mathbf{R}_{x}$ span the signal subspace $\mathfrak{R}_{s}$. In the presence of noise, we apply an $M$-rank approximation of $\mathbf{R}_{x}$ to estimate the bases of signal subspace. According to Lemma 7, we know that the best $M$-rank approximation of $\mathbf{R}_{x}$ has $4 M$ eigenvalues, thus we can use the eigenvectors $\mathbf{v}_{1}, \ldots, \mathbf{v}_{4 M}$ associated with the largest $4 M$ eigenvalues as the bases of signal subspaces. Denote $\mathbf{E}_{s}=\left[\mathbf{v}_{1}, \ldots, \mathbf{v}_{4 M}\right]$ and $\mathbf{E}_{n}=$ $\left[\mathbf{v}_{4 M}, \ldots, \mathbf{v}_{4 N}\right]$, then $\mathfrak{R}_{s}=\operatorname{span}\left(\mathbf{E}_{s}\right)$ and $\mathfrak{R}_{n}=\operatorname{span}\left(\mathbf{E}_{n}\right)$. Further, define $\mathbf{P}_{n}=\mathbf{E}_{n} \mathbf{E}_{n}^{H} \in \mathbb{U}_{\nVdash N}^{N \times N}$, we have $\left\|\mathbf{P}_{n} \mathbf{a}_{m}\right\|=0$, then

$$
\left\{\theta_{m}, \varphi_{m}\right\}=\arg \left\{\min _{\theta, \varphi, \gamma, \eta}\left\|\mathbf{P}_{n} \mathbf{a}_{\theta, \varphi, \gamma, \eta}\right\|\right\} .
$$

In the presence of finite data length, $\mathbf{R}_{x}$ can be estimated as follows:

$$
\widetilde{\mathbf{R}}_{x}=\frac{1}{L} \sum_{l=1}^{L} \mathbf{x}\left(t_{l}\right) \mathbf{x}^{H}\left(t_{l}\right)
$$

Accordingly, $\mathbf{E}_{n}$ and $\mathbf{P}_{n}$ can be estimated by eigendecomposing $\widetilde{\mathbf{R}}_{x}$.

A question of fundamental interests is whether the indetermination of quad-quaternion eigenvectors impacts the results of MUSIC. According to Lemma 8, the indetermination of eigenvectors does not impact the vector space spanned by them, and thus does not impact the estimation of noise subspace. Since the performance of MUSIC-like algorithms is mainly dependent on the accuracy of subspace estimation, the indetermination of eigenvectors does not impact the results of quad-quaternion MUSIC. 


\subsection{Decoupling of angular and polarization parameters}

According to (28), a $4 \mathrm{D}$ search is required for DOA estimation, which might be computationally prohibitive. We next discuss how to decouple polarization from DOA estimation for the purpose of reducing the computational burden. Firstly, we prove the following lemma.

Lemma 9. Given $\mathbf{h} \in\left(\mathbb{C}^{(L)}\right)^{M \times 1}, L \in\{i, j, k, I, J, K\}$ and a Hermitian matrix $\mathbf{F} \in\left(\mathbb{U}_{\mathbb{H}}\right)^{M \times M}$, then $S\left(\mathbf{h}^{H} \mathbf{F h}\right)=\mathbf{h}^{H} S(\mathbf{F}$ | $\{1, L\}) \mathbf{h}$. Here, $S\left(\mathbf{h}^{H} \mathbf{F h}\right)$ and $S(\mathbf{F} \mid\{1, L\})$ denote the scalar part of $\mathbf{h}^{H} \mathbf{F h}$ and the $\{1, L\}$-match of $\mathbf{F}$, respectively.

Proof. Without loss of generality, we assume $L=J$. Hence, $\mathbf{h} \in\left(\mathbb{C}^{(J)}\right)^{M \times 1}$. Let further $\mathbf{F}=\left(\mathbf{F}_{00}+I \mathbf{F}_{01}\right)+i\left(\mathbf{F}_{10}+I \mathbf{F}_{11}\right)+$ $j\left(\mathbf{F}_{20}+\mathbf{F}_{21}\right)+k\left(\mathbf{F}_{30}+\mathbf{F}_{31}\right)$, then $S(\mathbf{F} \mid\{1, J\})=\mathbf{F}_{00}$. Since $\mathbf{F}^{H}=\mathbf{F}$, we have $\mathbf{F}_{00}^{H}=\mathbf{F}_{00}$. Then it is further obtained that

$$
\begin{aligned}
S\left(\mathbf{h}^{H} \mathbf{F h}\right)= & S\left[\mathbf{h}^{H}\left(\mathbf{F}_{00}+I \mathbf{F}_{01}\right)+i\left(\mathbf{F}_{10}+I \mathbf{F}_{11}\right)\right. \\
& \left.+j\left(\mathbf{F}_{20}+\mathbf{F}_{21}\right)+k\left(\mathbf{F}_{30}+\mathbf{F}_{31}\right) \mathbf{h}\right] \\
= & S\left(\mathbf{h}^{H} \mathbf{F}_{00} \mathbf{h}\right)=\mathbf{h}^{H} \mathbf{F}_{00} \mathbf{h}=\mathbf{h}^{H} S(\mathbf{F} \mid\{1, J\}) \mathbf{h} .
\end{aligned}
$$

We use the above-mentioned lemma to discuss the decoupling of angular and polarization parameters. Let $\mathbf{u}=$ $\mathbf{P}_{n} \mathbf{a}_{\theta, \varphi, \gamma, \eta} \in \mathbb{H}_{\mathbb{H}}^{(N \times 1)}$, then

$$
\left\|\mathbf{P}_{n} \mathbf{a}_{\theta, \varphi, \gamma, \eta}\right\|=\sqrt{S\left(\mathbf{u}^{H} \mathbf{u}\right)} .
$$

According to (23), and denote $\boldsymbol{\Xi}_{\theta, \varphi}=\mathbf{P}_{n} \cdot \Theta_{\theta, \varphi} \otimes \mathbf{d}_{\theta, \varphi}$, then we have the following equation from Lemma 9:

$S\left(\mathbf{u}^{H} \mathbf{u}\right)=S\left(\mathbf{h}_{\gamma, \eta}^{H} \boldsymbol{\Xi}_{\theta, \varphi}^{H} \boldsymbol{\Xi}_{\theta, \varphi} \mathbf{h}_{\gamma, \eta}\right)=\mathbf{h}_{\gamma, \eta}^{H} S\left(\boldsymbol{\Xi}_{\theta, \varphi}^{H} \boldsymbol{\Xi}_{\theta, \varphi} \mid\{1, J\}\right) \mathbf{h}_{\gamma, \eta}$.

Note further that $\mathbf{h}_{\gamma, \eta}^{H} \mathbf{h}_{\gamma, \eta}=1$ and $S\left(\boldsymbol{\Xi}_{\theta, \varphi}^{H} \boldsymbol{\Xi}_{\theta, \varphi} \mid\{1, J\}\right)$ is a complex-valued Hermitian matrix, then according to the Rayleigh-Ritz theorem [11], we obtain

$$
\begin{aligned}
\min _{\theta, \varphi, \gamma, \eta}\left\|\mathbf{P}_{n} \mathbf{a}_{\theta, \varphi, \gamma, \eta}\right\| & =\min _{\theta, \varphi, \gamma, \eta}\left(\frac{\mathbf{h}_{\gamma, \eta}^{H} S\left(\boldsymbol{\Xi}_{\theta, \varphi}^{H} \boldsymbol{\Xi}_{\theta, \varphi} \mid\{1, J\}\right) \mathbf{h}_{\gamma, \eta}}{\mathbf{h}_{\gamma, \eta}^{H} \mathbf{h}_{\gamma, \eta}}\right) \\
& =\lambda_{\min }\left(S\left(\boldsymbol{\Xi}_{\theta, \varphi}^{H} \boldsymbol{\Xi}_{\theta, \varphi} \mid\{1, J\}\right)\right),
\end{aligned}
$$

where $\lambda_{\min }\left(S\left(\boldsymbol{\Xi}_{\theta, \varphi}^{H} \boldsymbol{\Xi}_{\theta, \varphi} \mid\{1, J\}\right)\right)$ denotes the smallest eigenvalue of $S\left(\boldsymbol{\Xi}_{\theta, \varphi}^{H} \boldsymbol{\Xi}_{\theta, \varphi} \mid\{1, J\}\right)$. Thus, the $4 \mathrm{D}$ search problem is reduced to a $2 \mathrm{D}$ search.

For clarity, we finally summarize the above split method (termed as QQ-MUSIC) as follows:

Step 1. calculate the sampled covariance matrix $\widetilde{\mathbf{R}}_{x}$ according to (29);

Step 2. apply the quad-quaternion EVD to $\widetilde{\mathbf{R}}_{x}$, select the $4(N-M)$ eigenvectors associated to the smallest $4(N-M)$ eigenvalues, and calculate the noise subspace projector $\mathbf{P}_{n}$;

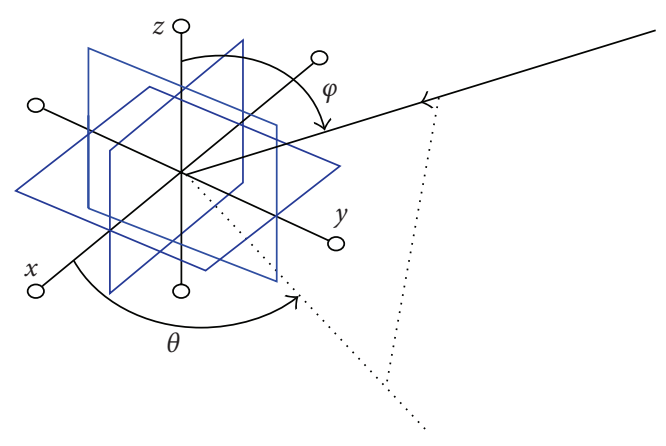

FIGURE 2: Coordinate system and angle definition.

Step 3. given an arbitrary $(\theta, \varphi) \in \mathfrak{I}_{1}$, calculate $\boldsymbol{\Xi}_{\theta, \varphi}=\mathbf{P}_{n}$. $\Theta_{\theta, \varphi} \otimes \mathbf{d}_{\theta, \varphi}$ and $S\left(\boldsymbol{\Xi}_{\theta, \varphi}^{H} \boldsymbol{\Xi}_{\theta, \varphi} \mid\{1, J\}\right)$.

Step 4. then the DOA estimates are obtained by

$$
\underset{(\theta, \varphi) \in \mathfrak{I}_{1}}{\arg \min }\left\{\lambda_{\min }\left(\mathbf{F}_{\theta, \varphi}\right)\right\} .
$$

It is important to note that QQ-MUSIC cannot fulfill simultaneous estimation of DOA and polarization. The problem of polarization estimation or joint DOApolarization estimation remains unresolved and is currently under investigation by the authors.

\subsection{Computation complexity}

In this section, the computational complexity of QQMUSIC, BQ-MUSIC, and long-vector MUSIC is addressed. As addressed in [36, 37], the covariance matrix estimation best illustrates the complexity difference of the three algorithms, therefore we only consider the computational complexity involved in this part. The evaluation of computational complexity includes two aspects: memory requirement and number of real number additions (A), multiplications (M), and divisions $(\mathrm{D})$.

Assume that the array comprises $N$ complete EM vector sensors, and $T$ snapshot vectors are available. The quadquaternion array output $\mathbf{X} \in\left(\mathbb{U}_{\mathbb{H}}\right)^{N \times T}$ then is given by

$$
\mathbf{X}=\mathbf{X}_{0}+I \mathbf{X}_{1}=\left(i \mathbf{X}_{01}+j \mathbf{X}_{02}+k \mathbf{X}_{03}\right)+I\left(i \mathbf{X}_{11}+j \mathbf{X}_{12}+k \mathbf{X}_{13}\right),
$$

where $\mathbf{X}_{0}, \mathbf{X}_{1} \in\left(\mathbb{I}_{\mathbb{C}^{(J)}}\right)^{N \times T}$, and $\mathbf{X}_{0 n}, \mathbf{X}_{1 n} \in\left(\mathbb{C}^{(J)}\right)^{N \times T}$, $n=1,2,3$. Then the biquaternion data model $\left(\mathbf{X}_{b} \in\right.$ $\left.\left(\mathbb{H}_{\mathbb{C}^{(J)}}\right)^{2 N \times T}\right)$ and the long-vector data model $\left(\mathbf{X}_{l v} \in\right.$ $\left(\mathbb{C}^{(J)}\right)^{6 N \times T}$ ) for the same array output are, respectively, written as

$$
\mathbf{X}_{b}=\left[\mathbf{X}_{0}^{T}, \mathbf{X}_{1}^{T}\right]^{T}, \quad \mathbf{X}_{l v}=\left[\mathbf{X}_{01}^{T}, \mathbf{X}_{02}^{T}, \mathbf{X}_{03}^{T}, \mathbf{X}_{11}^{T}, \mathbf{X}_{12}^{T}, \mathbf{X}_{13}^{T}\right]^{T} .
$$

Moreover, the sampled covariance matrices in the three models can be calculated as follows:

$$
\widetilde{\mathbf{R}}_{\mathrm{Q}}=\frac{1}{T} \mathbf{X X}^{H}, \quad \widetilde{\mathbf{R}}_{\mathrm{B}}=\frac{1}{T} \mathbf{X}_{b} \mathbf{X}_{b}^{H}, \quad \widetilde{\mathbf{R}}_{\mathrm{LV}}=\frac{1}{T} \mathbf{X}_{l v} \mathbf{X}_{l v}^{H},
$$


TABLE 2: Computational effort for covariance estimation.

\begin{tabular}{lcccc}
\hline & Memory requirements (complex values) & Real multiplications & Real additions & Real divisions \\
\hline QQ-MUSIC & $8 N^{2}$ & $256 N^{2} T$ & $(256 T-16) N^{2}$ & $16 N^{2}(D)$ \\
BQ-MUSIC & $16 N^{2}$ & $256 N^{2} T$ & $(256 T-32) N^{2}$ & $32 N^{2}{ }_{(D)}$ \\
LV-MUSIC & $36 N^{2}$ & $144 N^{2} T$ & $(144 T-72) N^{2}$ & $72 N^{2}{ }_{(D)}$ \\
\hline
\end{tabular}

where $\widetilde{\mathbf{R}}_{\mathrm{Q}}, \widetilde{\mathbf{R}}_{B}, \widetilde{\mathbf{R}}_{\mathrm{LV}}$ are sampled covariance matrices used in QQ-MUSIC, BQ-MUSIC, and LV-MUSIC, respectively.

From (37), $\widetilde{\mathbf{R}}_{\mathrm{Q}}$ has $N^{2}$ entries, each of which is quadquaternion valued and can be represented by eight complex numbers. Therefore, a memory of at least $8 N^{2}$ complex numbers is required in the quad-quaternion case. Similarly, for biquaternion and long-vector models, $16 N^{2}$ and $36 N^{2}$ complex numbers are required, respectively.

Let us now evaluate the total number of basic arithmetic operations needed for estimation of the covariance matrix. As revealed by (37), every entry of $\widetilde{\mathbf{R}}_{\mathrm{Q}}$ is obtained by $T$ quad-quaternion multiplications, $T-1$ quad-quaternion additions, and a division by a real value. Note that one quadquaternion multiplication implies $16^{2}$ real multiplications plus $16 \times 15$ real additions, one quad-quaternion addition implies 16 real additions, and the division by a real value equals 16 real divisions. The number of operations needed for one entry is $\left[16_{(M)}^{2}+16 \times 15_{(A)}\right] T+16(T-1)_{(A)}+16_{(D)}$, where subscripts “ $(M)$," “ $(A)$," " $(D)$ " denote real multiplication, real addition, and real division, respectively. Thus, the total number is $\left\{\left[16_{(M)}^{2}+16 \times 15_{(A)}\right] T+16(T-1)_{(A)}+16_{(D)}\right\}$. $N^{2}=256 N^{2} T_{(M)}+(256 T-16) N_{(A)}^{2}+16 N_{(D)}^{2}$. Similarly, the total numbers of arithmetic operations in biquaternion and long-vector models are given by $256 N^{2} T_{(M)}+(256 \mathrm{~T}-$ $32) N_{(A)}^{2}+32 N_{(D)}^{2}$ and $144 N^{2} T_{(M)}+(144 T-72) N_{(A)}^{2}+$ $72 N_{(D)}^{2}$, respectively. Table 2 summarizes the covariance matrix computational efforts for all the three algorithms. We can see that QQ-MUSIC largely reduces the memory requirements, mainly due to the more economical formulism of quad-quaternion model. In addition, with regard to basic arithmetic operation number, we can see that QQMUSIC requires $16 N^{2}$ less real divisions and $16 N^{2}$ more real additions than $\mathrm{BQ}-\mathrm{MUSIC}$. Since the computational complexity of divisions is much more than that of additions, QQ-MUSIC slightly outperforms BQ-MUSIC in this aspect. We may also note that LV-MUSIC requires least operations for estimating the covariance matrix, which conflicts our intuition that a more concise model should lead to less computational complexity. This fact can be explained as follows. In QQ-MUSIC, we are using a 16D algebra to model six-component vector sensors, and only twelve imaginary units of quad-quaternions are used in this formulation. Therefore, this insufficient use of quad-quaternions results in more arithmetic operations.

\subsection{Orthogonality-measure comparison}

As addressed in [37], vector orthogonality in higher dimensional algebra imposes stronger constraints on vector components. In this part, we take a further look into the quadquaternion-related orthogonality.
Consider two quad-quaternion vectors $\mathbf{x}, \mathbf{y} \in\left(\mathbb{Q}_{\mathbb{H}}\right)^{N_{x} \times 1}$ given by

$$
\begin{aligned}
& \mathbf{x}=\left(\mathbf{x}_{01}+I \mathbf{x}_{11}\right) i+\left(\mathbf{x}_{02}+I \mathbf{x}_{12}\right) j+\left(\mathbf{x}_{03}+I \mathbf{x}_{13}\right) k, \\
& \mathbf{y}=\left(\mathbf{y}_{01}+I \mathbf{y}_{11}\right) i+\left(\mathbf{y}_{02}+I \mathbf{y}_{12}\right) j+\left(\mathbf{y}_{03}+I \mathbf{y}_{13}\right) k .
\end{aligned}
$$

The corresponding biquaternion representation and complex representation then can be written as

$$
\begin{aligned}
& \mathbf{x}_{b q}=\left[\begin{array}{ll}
\mathbf{x}_{01}^{T}, & \mathbf{x}_{11}^{T}
\end{array}\right]^{T} i+\left[\begin{array}{ll}
\mathbf{x}_{02}^{T}, & \mathbf{x}_{12}^{T}
\end{array}\right]^{T} j+\left[\mathbf{x}_{03}^{T}, \mathbf{x}_{13}^{T}\right]^{T}, \\
& k \in\left(\mathbb{U}_{\mathbb{C}}\right)^{2 N_{x} \times 1}, \\
& \mathbf{y}_{b q}=\left[\mathbf{y}_{01}^{T}, \mathbf{y}_{11}^{T}\right]^{T} i+\left[\mathbf{y}_{02}^{T}, \mathbf{y}_{12}^{T}\right]^{T} j+\left[\mathbf{y}_{03}^{T}, \mathbf{y}_{13}^{T}\right]^{T}, \\
& k \in\left(\mathbb{U}_{\mathbb{C}}\right)^{2 N_{x} \times 1},
\end{aligned}
$$

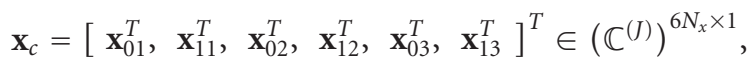

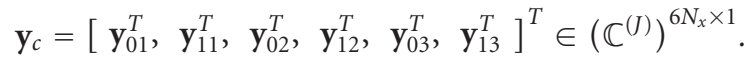

Imposing the orthogonal constraint on quad-quaternion vectors $\left(\mathbf{x}^{H} \mathbf{y}=0\right)$ yields

$$
\begin{aligned}
& \mathbf{x}_{01}^{H} \mathbf{y}_{01}+ \mathbf{x}_{11}^{H} \mathbf{y}_{11}+\mathbf{x}_{02}^{H} \mathbf{y}_{02}+\mathbf{x}_{12}^{H} \mathbf{y}_{12}+\mathbf{x}_{03}^{H} \mathbf{y}_{03}+\mathbf{x}_{13}^{H} \mathbf{y}_{13}=0, \\
& \mathbf{x}_{01}^{T} \mathbf{y}_{11}- \mathbf{x}_{11}^{T} \mathbf{y}_{01}+\mathbf{x}_{02}^{T} \mathbf{y}_{12}-\mathbf{x}_{12}^{T} \mathbf{y}_{02}+\mathbf{x}_{03}^{T} \mathbf{y}_{13}-\mathbf{x}_{13}^{T} \mathbf{y}_{03}=0, \\
& \mathbf{x}_{02}^{H} \mathbf{y}_{03}+\mathbf{x}_{12}^{H} \mathbf{y}_{13}-\mathbf{x}_{03}^{H} \mathbf{y}_{02}-\mathbf{x}_{13}^{H} \mathbf{y}_{12}=0, \\
& \mathbf{x}_{02}^{T} \mathbf{y}_{13}-\mathbf{x}_{12}^{T} \mathbf{y}_{03}-\mathbf{x}_{03}^{T} \mathbf{y}_{12}+\mathbf{x}_{13}^{T} \mathbf{y}_{02}=0, \\
& \mathbf{x}_{03}^{H} \mathbf{y}_{01}+\mathbf{x}_{13}^{H} \mathbf{y}_{11}-\mathbf{x}_{01}^{H} \mathbf{y}_{03}-\mathbf{x}_{11}^{H} \mathbf{y}_{13}=0, \\
& \mathbf{x}_{03}^{T} \mathbf{y}_{11}-\mathbf{x}_{13}^{T} \mathbf{y}_{01}-\mathbf{x}_{01}^{T} \mathbf{y}_{13}+\mathbf{x}_{11}^{T} \mathbf{y}_{03}=0, \\
& \mathbf{x}_{01}^{H} \mathbf{y}_{02}+\mathbf{x}_{11}^{H} \mathbf{y}_{12}-\mathbf{x}_{02}^{H} \mathbf{y}_{01}-\mathbf{x}_{12}^{H} \mathbf{y}_{11}=0, \\
& \mathbf{x}_{01}^{T} \mathbf{y}_{12}-\mathbf{x}_{11}^{T} \mathbf{y}_{02}-\mathbf{x}_{02}^{T} \mathbf{y}_{11}+\mathbf{x}_{12}^{T} \mathbf{y}_{01}=0 .
\end{aligned}
$$

In contrast, orthogonal constraint on biquaternion vectors $\left(\mathbf{x}_{b q}^{H} \mathbf{y}_{b q}=0\right)$ results in

$$
\begin{aligned}
\mathbf{x}_{01}^{H} \mathbf{y}_{01}+ & \mathbf{x}_{11}^{H} \mathbf{y}_{11}+\mathbf{x}_{02}^{H} \mathbf{y}_{02}+\mathbf{x}_{12}^{H} \mathbf{y}_{12}+\mathbf{x}_{03}^{H} \mathbf{y}_{03}+\mathbf{x}_{13}^{H} \mathbf{y}_{13}=0, \\
& \mathbf{x}_{02}^{H} \mathbf{y}_{03}+\mathbf{x}_{12}^{H} \mathbf{y}_{13}-\mathbf{x}_{03}^{H} \mathbf{y}_{02}-\mathbf{x}_{13}^{H} \mathbf{y}_{12}=0, \\
& \mathbf{x}_{03}^{H} \mathbf{y}_{01}+\mathbf{x}_{13}^{H} \mathbf{y}_{11}-\mathbf{x}_{01}^{H} \mathbf{y}_{03}-\mathbf{x}_{11}^{H} \mathbf{y}_{13}=0, \\
& \mathbf{x}_{01}^{H} \mathbf{y}_{02}+\mathbf{x}_{11}^{H} \mathbf{y}_{12}-\mathbf{x}_{02}^{H} \mathbf{y}_{01}-\mathbf{x}_{12}^{H} \mathbf{y}_{11}=0 .
\end{aligned}
$$

Moreover, the orthogonal constraint on complex vectors $\left(\mathbf{x}_{c}^{H} \mathbf{y}_{c}=0\right)$ leads to

$$
\mathbf{x}_{01}^{H} \mathbf{y}_{01}+\mathbf{x}_{11}^{H} \mathbf{y}_{11}+\mathbf{x}_{02}^{H} \mathbf{y}_{02}+\mathbf{x}_{12}^{H} \mathbf{y}_{12}+\mathbf{x}_{03}^{H} \mathbf{y}_{03}+\mathbf{x}_{13}^{H} \mathbf{y}_{13}=0 .
$$




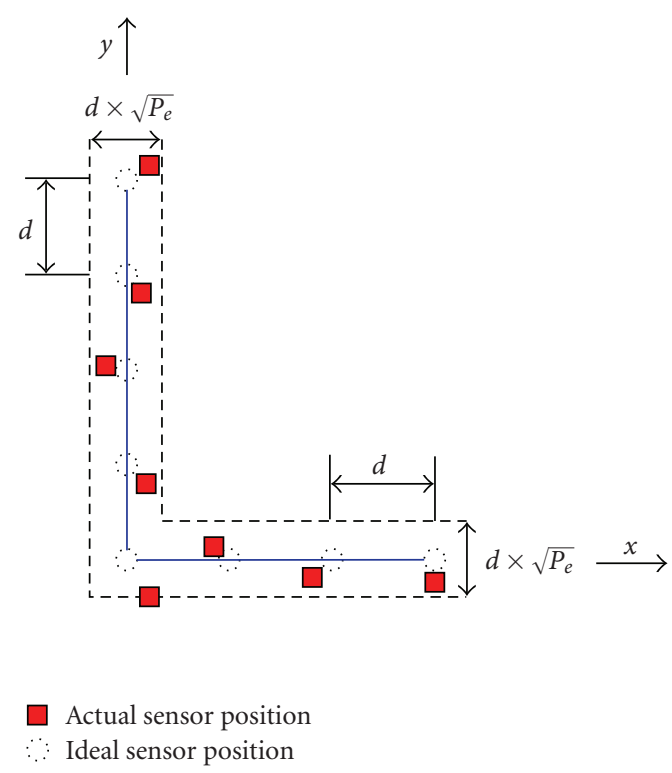

FIGURE 3: An array with sensor position errors.

By comparing (40), (41) and (42), it is obtained that:

$$
\mathbf{x}^{H} \mathbf{y}=0 \Longrightarrow \mathbf{x}_{b q}^{H} \mathbf{y}_{b q}=0 \Longrightarrow \mathbf{x}_{c}^{H} \mathbf{y}_{c}=0
$$

Consequently, the quad-quaternion orthogonality can impose stronger constraints than both biquaternion and complex algebra do. This property of quad-quaternions results in a better robustness of QQ-MUSIC to model errors, as to be demonstrated in Section 4.

\section{SIMULATION RESULTS}

In this section, simulation results are provided to compare the proposed QQ-MUSIC with both biquaternion-based (such as BQ-MUSIC) and complex-based methods (such as LV-MUSIC) for six-component EM vector-sensor arrays. It should be noted that BQ-MUSIC was actually proposed for three-component vector-sensor arrays [37]. Therefore, we here use a $2 \times 1$ biquaternion vector to represent a six-component vector sensor, and further we concatenate these vectors into a biquaternion long-vector to enable BQMUSIC.

We compare the proposed QQ-MUSIC with BQ-MUSIC, LV-MUSIC, and polarimetric smoothing algorithm (PSAMUSIC [30]), in terms of robustness to model errors and DOA estimation performance under different levels of signal-to-noise ratio (SNR). All the statistics shown here are computed by averaging the results of 200 independent trials. The array used here is an L-shaped array that comprises four and five EM vector sensors along the $x$-axis and $y$-axis, respectively (see Figure 3 ). The spacing between two adjacent EM vector sensors is $d=\lambda / 2$. Before representing the results, we introduce the following two model errors.

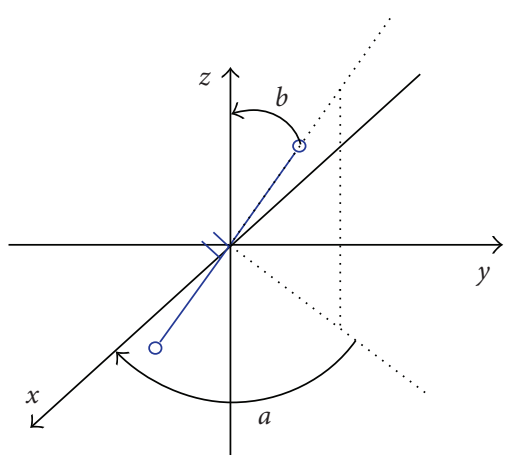

(a)

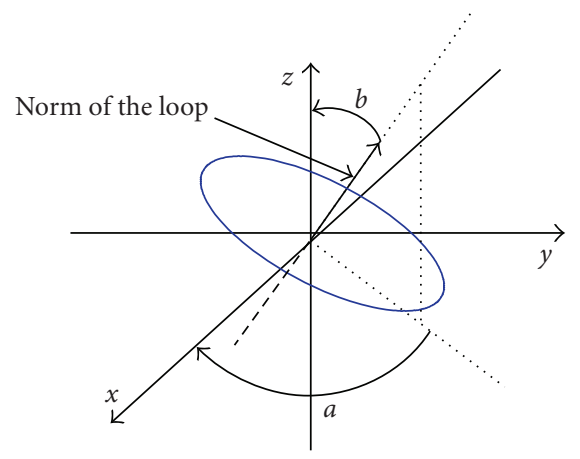

(b)

FIGURE 4: A short dipole or loop with arbitrary orientation.

\section{Sensor-position error}

the positions of EM vector sensors are not precisely known. In the simulations, we model such sensor position error by additive uniformly distributed noise, that is,

$$
\overline{\boldsymbol{k}}_{n}=\mathbf{k}_{n}+\sqrt{P_{e}} \cdot d \cdot\left[\varepsilon_{x}, \varepsilon_{y}, 0\right]^{T}
$$

where $\overline{\boldsymbol{k}}_{n}$ and $\mathbf{k}_{n}$ are the actual and ideal position coordinates of the $n$th EM vector sensor, respectively, $\varepsilon_{x}$ and $\varepsilon_{y}$ are uniformly distributed noise terms, and $P_{e}$ is the power of sensor position error.

\section{Sensor-orientation error}

the orientation angles of a dipole and a loop are illustrated in Figure 4. With an orientation angle $(\alpha, \beta)$, where $\alpha \in$ $[0,2 \pi), \beta \in[0, \pi / 2]$, the outputs of a dipole and a loop are, respectively, given by

$$
\begin{aligned}
E_{\alpha, \beta}^{(\theta, \varphi, \gamma, \eta)}= & {[\cos \alpha \sin \beta, \sin \alpha \sin \beta, \cos \beta] } \\
& \cdot\left[E_{x}^{(\theta, \varphi, \gamma, \eta)}, E_{y}^{(\theta, \varphi, \gamma, \eta)}, E_{z}^{(\theta, \varphi, \gamma, \eta)}\right] T, \\
H_{\alpha, \beta}^{(\theta, \varphi, \gamma, \eta)}= & {[\cos \alpha \sin \beta, \sin \alpha \sin \beta, \cos \beta] } \\
& \cdot\left[H_{x}^{(\theta, \varphi, \gamma, \eta)}, H_{y}^{(\theta, \varphi, \gamma, \eta)}, H_{z}^{(\theta, \varphi, \gamma, \eta)}\right] T,
\end{aligned}
$$

where $E_{x}^{(\theta, \varphi, \gamma, \eta)}, E_{y}^{(\theta, \varphi, \gamma, \eta)}, E_{z}^{(\theta, \varphi, \gamma, \eta)}$, and $H_{x}^{(\theta, \varphi, \gamma, \eta)}, H_{y}^{(\theta, \varphi, \gamma, \eta)}$, $H_{z}^{(\theta, \varphi, \gamma, \eta)}$ are given in (23). Let the orientation angles of 
the three dipoles of the $n$th EM vector sensor be $\left(\bar{\alpha}_{1, n}, \bar{\beta}_{1, n}\right)$, $\left(\bar{\alpha}_{2, n}, \bar{\beta}_{2, n}\right)$, and $\left(\bar{\alpha}_{3, n}, \bar{\beta}_{3, n}\right)$, while the orientation angles of the three loops be $\left(\bar{\alpha}_{4, n}, \bar{\beta}_{4, n}\right),\left(\bar{\alpha}_{5, n}, \bar{\beta}_{5, n}\right)$, and $\left(\bar{\alpha}_{6, n}, \bar{\beta}_{6, n}\right)$, then we have

$$
\begin{array}{r}
{\left[\bar{\alpha}_{l, n}, \bar{\beta}_{l, n}\right]=\left[\alpha_{l}, \beta_{l}\right]+\sqrt{P_{e}}\left[\varepsilon_{\alpha, l, n}, \varepsilon_{\beta, l, n}\right]} \\
l=1, \ldots, 6 ; n=1, \ldots, N
\end{array}
$$

where $P_{e}$ is the power of the sensor orientation error, $\varepsilon_{\alpha, l, n}, \varepsilon_{\beta, l, n}$ are uniformly distributed noise terms, $\left(\alpha_{1}, \beta_{1}\right)=$ $\left(\alpha_{4}, \beta_{4}\right)=(0, \pi / 2),\left(\alpha_{2}, \beta_{2}\right)=\left(\alpha_{5}, \beta_{5}\right)=(\pi / 2, \pi / 2)$, $\left(\alpha_{3}, \beta_{3}\right)=\left(\alpha_{6}, \beta_{6}\right)=(0,0)$ are the corresponding nominal orientation angles in the absence of sensor orientation error. Combining (22), (23), (45), and (46), the output of the $n$th EM vector sensor equals

$$
\begin{aligned}
\bar{p}_{\theta, \varphi, \gamma, \eta}^{(n)}=\{ & \left(\begin{array}{c}
\cos \varepsilon_{\beta, 1, n} \sin \left(\varepsilon_{\alpha, 1, n}-\varphi\right)-I \cdot\left(\sin \varepsilon_{\beta, 4, n} \sin \theta-\cos \left(\varepsilon_{\alpha, 4, n}-\varphi\right) \cos \varepsilon_{\beta, 4, n} \cos \theta\right) \\
\cos \varepsilon_{\beta, 1, n} \cos \theta \cos \left(\varepsilon_{\alpha, 1, n}-\varphi\right)+\sin \varepsilon_{\beta, 1, n} \sin \theta+I \cdot \cos \varepsilon_{\beta, 4, n} \sin \left(\varepsilon_{\alpha, 4, n}-\varphi\right)
\end{array}\right)^{T} \cdot i \\
& +\left(\begin{array}{c}
\cos \varepsilon_{\beta, 2, n} \cos \left(\varepsilon_{\alpha, 2, n}-\varphi\right)-I \cdot\left(\sin \varepsilon_{\beta, 5, n} \sin \theta-\sin \left(\varepsilon_{\alpha, 5, n}-\varphi\right) \cos \varepsilon_{\beta, 5, n} \cos \theta\right) \\
\cos \varepsilon_{\beta, 2, n} \cos \theta \sin \left(\varphi-\varepsilon_{1,2, n}\right)+\sin \varepsilon_{\beta, 2, n} \sin \theta+I \cdot \cos \varepsilon_{\beta, 5, n} \cos \left(\varepsilon_{\alpha, 5, n}-\varphi\right)
\end{array}\right)^{T} \cdot j \\
& \left.+\left(\begin{array}{c}
\sin \varepsilon_{\beta, 3, n} \sin \left(\varepsilon_{\alpha, 3, n}-\varphi\right)+I \cdot\left(\cos \varepsilon_{\beta, 6, n} \sin \theta-\cos \left(\varepsilon_{\alpha, 6, n}-\varphi\right) \sin \varepsilon_{\beta, 6, n} \cos \theta\right) \\
\sin \varepsilon_{\beta, 3, n} \cos \theta \cos \left(\varepsilon_{\alpha, 3, n}-\varphi\right)-\cos \varepsilon_{\beta, 3, n} \sin \theta+I \cdot \sin \varepsilon_{\beta, 6, n} \sin \left(\varepsilon_{\alpha, 6, n}-\varphi\right)
\end{array}\right)^{T} \cdot k\right\} \cdot \mathbf{h}_{\gamma, \eta} \cdot
\end{aligned}
$$

Accordingly, the quad-quaternion expressions of the steering vector and the array output can be, respectively, modified as

$$
\begin{gathered}
\overline{\boldsymbol{a}}_{\theta, \varphi, \gamma, \eta}=\left[\bar{p}_{\theta, \varphi, \gamma, \eta}^{(1)} e^{J \cdot 2 \pi\left(\mathbf{k}_{1}^{T} \mathbf{e}_{\theta, \varphi} / \lambda\right)}, \ldots, \bar{p}_{\theta, \varphi, \gamma, \eta}^{(N)} e^{J \cdot 2 \pi\left(\mathbf{k}_{N}^{T} \mathbf{e}_{\theta, \varphi} / \lambda\right)}\right] T, \\
\overline{\boldsymbol{x}}(t)=\sum_{m=1}^{M} \overline{\boldsymbol{a}}_{\theta_{m}, \varphi_{m}, \gamma_{m}, \eta_{m}} \boldsymbol{s}_{m}(t)+\mathbf{n}(t) .
\end{gathered}
$$

In the first experiment, we assume that only the sensor position error exists. Three uncorrelated signals are from $\left(\theta_{1}, \varphi_{1}\right)=\left(8^{\circ}, 90^{\circ}\right),\left(\theta_{2}, \varphi_{2}\right)=\left(35^{\circ}, 90^{\circ}\right)$, and $\left(\theta_{3}, \varphi_{3}\right)=$ $\left(60^{\circ}, 90^{\circ}\right)$ (to exclude the effect of DOA ambiguity on the comparison, we here only consider azimuth angle estimation), respectively, with polarizations $\left(\gamma_{1}, \eta_{1}\right)=\left(45^{\circ}, 0^{\circ}\right)$, $\left(\gamma_{2}, \eta_{2}\right)=\left(45^{\circ}, 90^{\circ}\right)$, and $\left(\gamma_{3}, \eta_{3}\right)=\left(45^{\circ}, 180^{\circ}\right)$, respectively. The sensor noise is assumed to be Gaussian white and uncorrelated with the incident signals. The overall root mean square error (RMSE) performance measure used here is defined as follows:

$$
\varepsilon=\frac{1}{M} \sum_{m=1}^{M} \sqrt{\frac{1}{N} \sum_{n=1}^{N_{s}}\left[\left(\theta_{m}-\tilde{\theta}_{n, m}\right)^{2}\right]}
$$

where $\tilde{\theta}_{n, m}$ is the estimate of true azimuth angle $\theta_{m}$ in the $n$th trial. It is plotted in Figure 5 that the curves of overall RMSE against sensor position error power for QQ-MUSIC, BQ-MUSIC, LV-MUSIC, and PSA-MUSIC wherein the SNR and the number of snapshots are fixed as $30 \mathrm{~dB}$ and 1000 , respectively. It can be seen that QQ-MUSIC provides the best estimation accuracy in the presence of sensor position error.

In the second experiment, we assume that only the sensor orientation error exists. The DOAs and polarizations of the incident signals are the same as the first example. The overall RMSE curves of QQ-MUSIC, BQ-MUSIC, LV-MUSIC, and

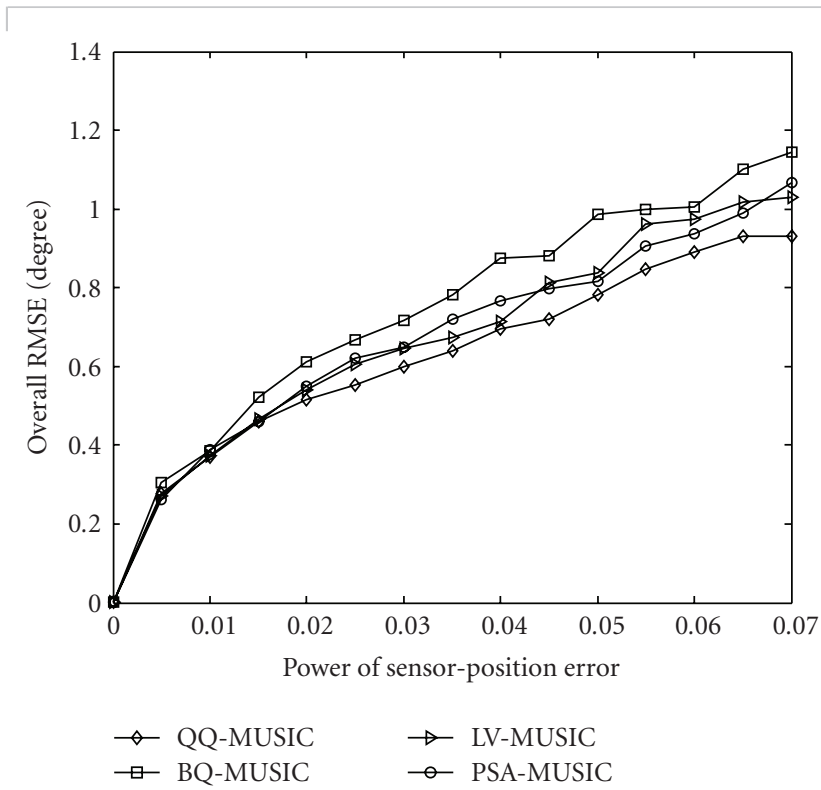

FIGURE 5: RMS estimation errors versus sensor position error power.

PSA-MUSIC against the power of sensor orientation error are plotted in Figure 6, wherein the SNR is constantly $30 \mathrm{~dB}$. We can see that QQ-MUSIC shows better robustness to sensor orientation error than BQ-MUSIC and LV-MUSIC. In particular, when the power of sensor orientation error is high, QQ-MUSIC can still provide reliable DOA estimates. It can also be observed that the performance of PSA-MUSIC is independent of the senor orientation error. This can be explained by noting that PSA-MUSIC does not preserve the polarization information, and thus is independent of the model error in the polarization dimension. 


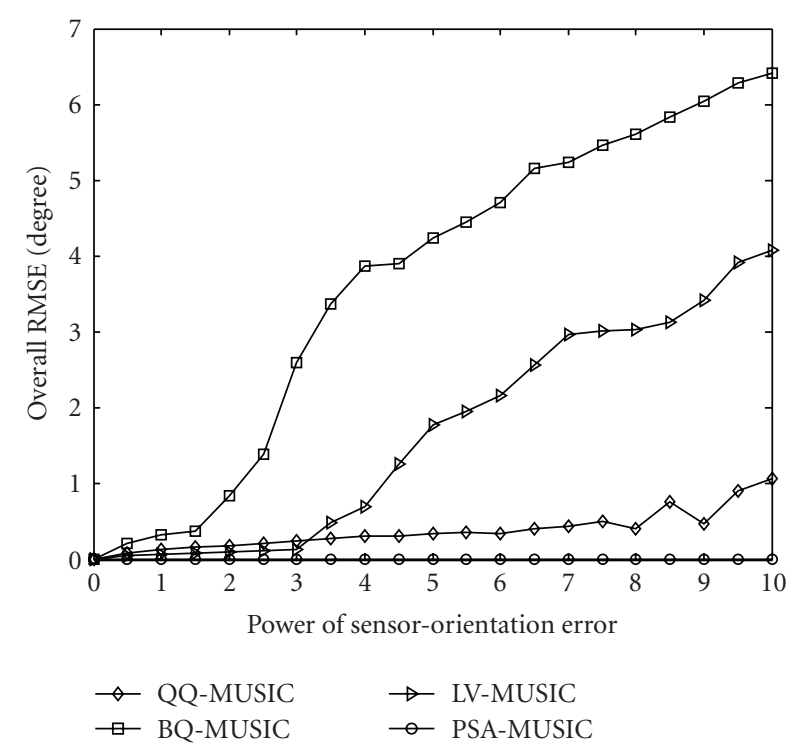

Figure 6: RMS estimation errors versus the power of sensor orientation error.

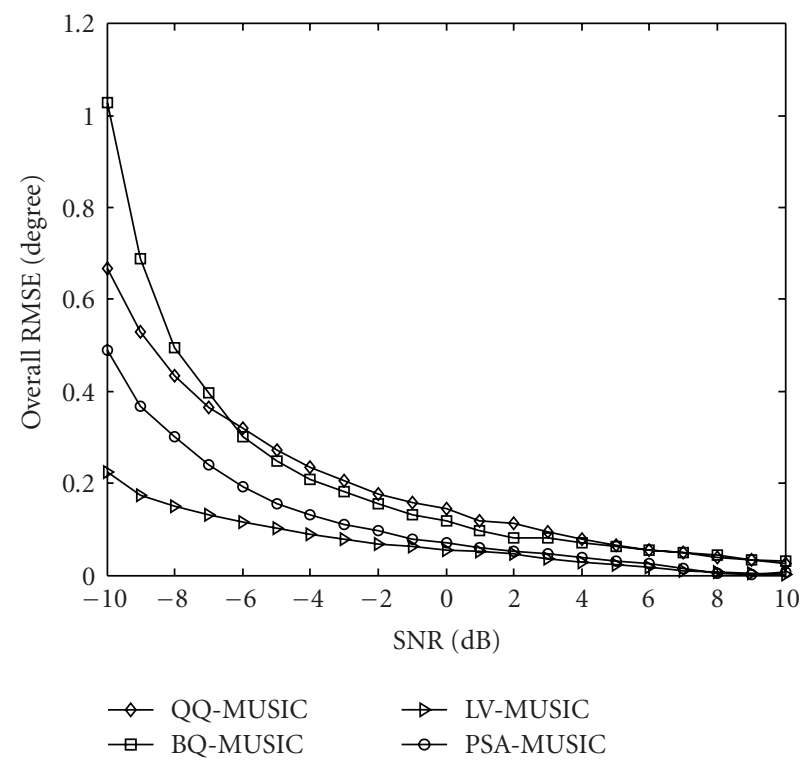

FIGURE 7: RMS estimation errors versus SNR for an ideal EM vector-sensor array.

We next compare the performance of QQ-MUSIC with BQ-MUSIC, LV-MUSIC, and PSA-MUSIC with respect to $\mathrm{SNR}$. The DOAs and polarizations are the same as the first two examples. The SNR is varied between $-10 \mathrm{~dB}$ and $10 \mathrm{~dB}$. Three cases are considered (1) in the absence of model error; (2) in the presence of sensor position error only, and the error power is $P_{e}=0.05$; (3) in the presence of sensor orientation error only, with model error power $P_{e}=5$. The results are given in Figures 7-9. From Figure 7, in a model error-free case, QQ-MUSIC and BQ-MUSIC are a bit inferior to LV-MUSIC and PSA-MUSIC. From Figure 8, we can see that QQ-MUSIC outperforms BQ-MUSIC, LV-

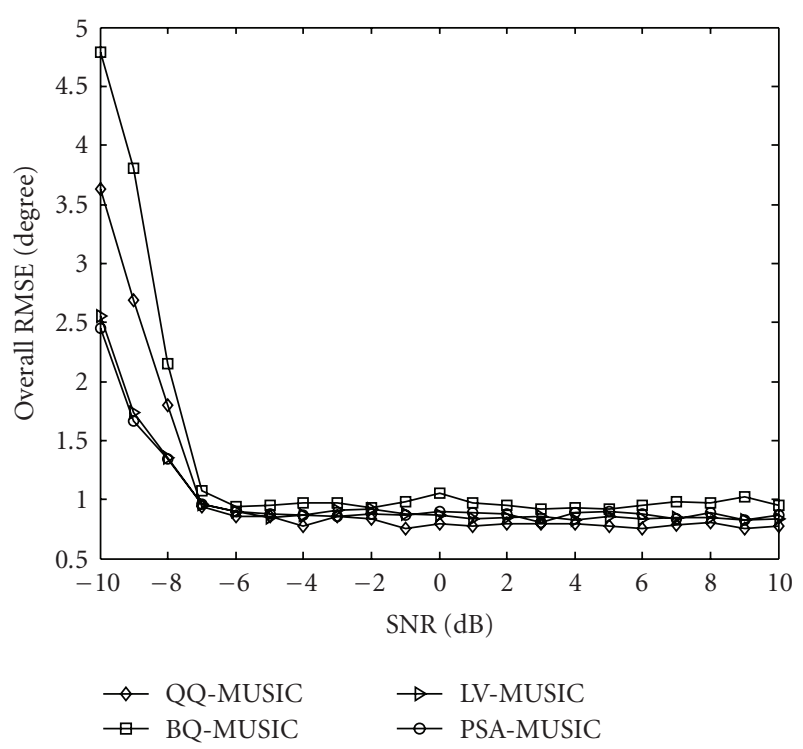

FIGURE 8: RMS estimation errors versus SNR in the presence of sensor position errors.

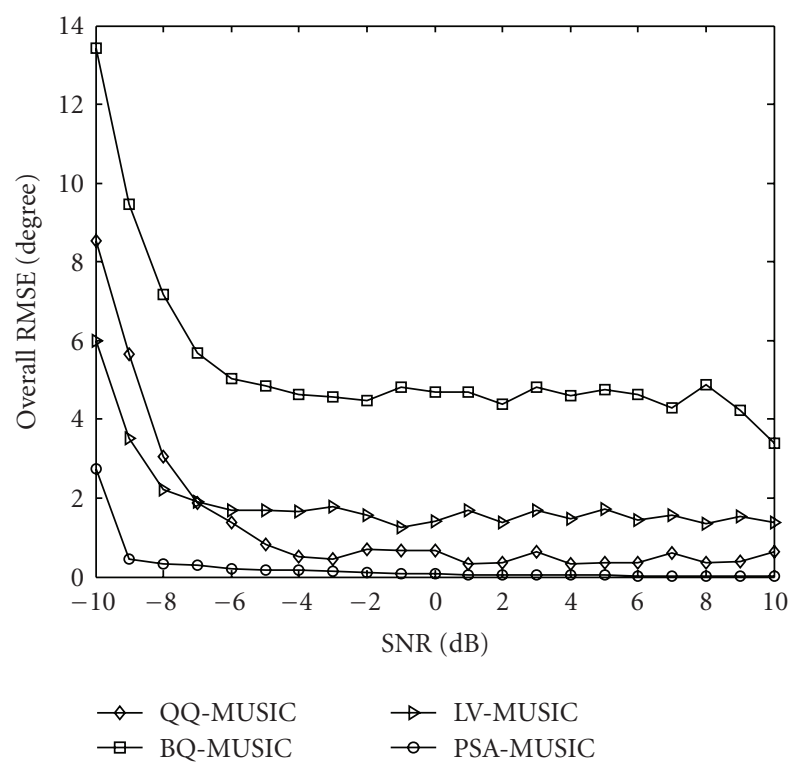

FIGURE 9: RMS estimation errors versus SNR in the presence of sensor orientation errors.

MUSIC, and PSA-MUSIC at all levels of SNR, in the presence of sensor position errors; from Figure 9, we see that QQ-MUSIC outperforms LV-MUSIC and PSA-MUSIC in sensor orientation errors, while slightly underperforms PSAMUSIC because the performance of the latter is independent of sensor orientation errors.

Then, we consider the case of several incident signals with close DOAs and polarizations. An "L"-shaped array with 20 complete EM vector sensors is used, and six incident signals are assumed to be impinging, among which the first and the second signals, the third and the fourth signals, and the fifth and the sixth signals are close to each other in both 


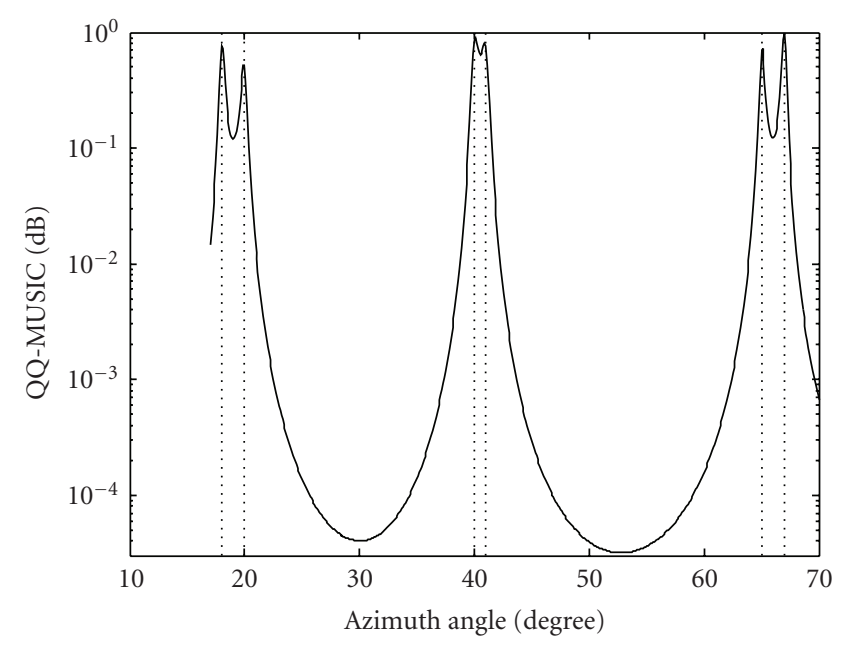

FIgURE 10: QQ-MUSIC spectrum in the presence of several close signals; $\mathrm{SNR}=30 \mathrm{~dB}$, the number of snapshots is 100 .

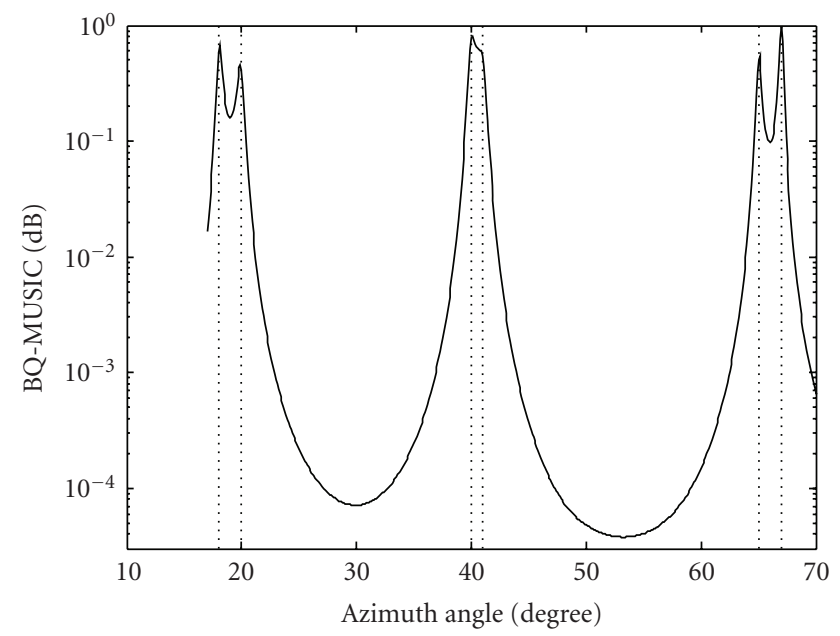

FIGURE 11: BQ-MUSIC spectrum in the presence of several close signals; $\mathrm{SNR}=30 \mathrm{~dB}$, the number of snapshots is 100 .

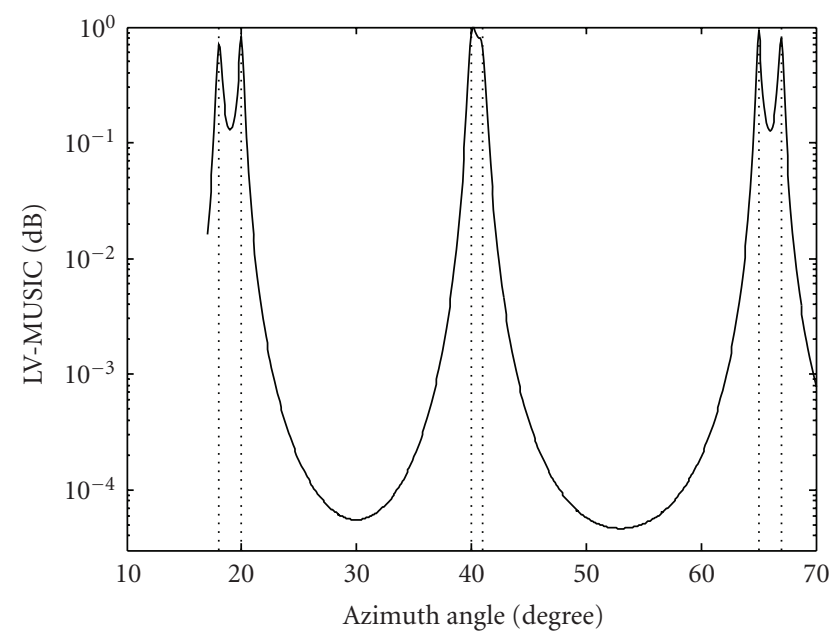

FIgURE 12: LV-MUSIC spectrum in the presence of several close signals; $\mathrm{SNR}=30 \mathrm{~dB}$, the number of snapshots is 100 . angular and polarization domains: $\left(\theta_{1}, \varphi_{1}\right)=\left(18^{\circ}, 90^{\circ}\right)$, $\left(\theta_{2}, \varphi_{2}\right)=\left(20^{\circ}, 90^{\circ}\right),\left(\theta_{3}, \varphi_{3}\right)=\left(40^{\circ}, 90^{\circ}\right),\left(\theta_{4}, \varphi_{4}\right)=$ $\left(41^{\circ}, 90^{\circ}\right),\left(\theta_{5}, \varphi_{5}\right)=\left(65^{\circ}, 90^{\circ}\right)$, and $\left(\theta_{6}, \varphi_{6}\right)=\left(67^{\circ}, 90^{\circ}\right)$; $\left(\gamma_{1}, \eta_{1}\right)=\left(\gamma_{2}, \eta_{2}\right)=\left(\gamma_{3}, \eta_{3}\right)=\left(\gamma_{4}, \eta_{4}\right)=\left(\gamma_{5}, \eta_{5}\right)=$ $\left(\gamma_{6}, \eta_{6}\right)=\left(0^{\circ}, 0^{\circ}\right)$ (to exclude the effect of DOA ambiguity on the comparison, we here only consider azimuth angle estimation). In addition, we assume that no model error exists for the above-mentioned array, the SNR is $30 \mathrm{~dB}$, and the number of snapshots is 100 . The spectra of QQMUSIC, BQ-MUSIC, and LV-MUSIC are given in Figures 11-12, in which the dotted lines indicate the true azimuth angles. We note that both BQ-MUSIC and LV-MUSIC fail to distinguish the third and the fourth signals, while QQMUSIC can successfully distinguish all the six sources with considerable accuracy.

\section{CONCLUSIONS}

In this paper, we have proposed a new DOA estimator, termed as quad-quaternion MUSIC (QQ-MUSIC), for sixcomponent EM vector-sensor arrays. This new MUSIC variant has employed a newly developed quad-quaternion model that provides a less memory-consuming way to deal with six-component EM vector-sensor outputs. Moreover, with sensor position error or sensor orientation error present, QQ-MUSIC has been shown to be able to offer better DOA estimation accuracy than both biquaternion-MUSIC (BQMUSIC) and long-vector MUSIC (LV-MUSIC). Thus, QQMUSIC may be more attractive than the examined methods in many practical situations, where model errors cannot be ignored.

\section{APPENDIX \\ PROOFS OF LEMMAS 5-8 IN SECTION 2}

Proof of Lemma 5. If we denote $\operatorname{conj}\left(\mathbf{Q} \mid \Gamma_{3}\right)=\operatorname{conj}((\operatorname{conj}(\mathbf{Q}$ $\left.\left.\left.\mid \Gamma_{1}\right)\right) \mid \Gamma_{2}\right)$, then by definition, $\Gamma_{3}$ is a set consisting of the bases whose coefficients are not inversed. Obviously, the coefficients of bases in $\Gamma_{1} \cap \Gamma_{2}$ are not inversed, and the coefficients of bases in $\left(\Gamma_{1} \cup \Gamma_{2}\right)^{\perp}$ are inversed twice so that they are also not inversed. Therefore, $\Gamma_{3}=\left(\Gamma_{1} \cap \Gamma_{2}\right) \cup$ $\left(\Gamma_{1} \cup \Gamma_{2}\right)^{\perp}$.

Proof of Lemma 6. Without loss of generality, here we only consider the case of right rank. Assume the right rank of $\mathbf{P} \in\left(\mathbb{H}_{\mathbb{H}}\right)^{M \times N}$ is $R$, then by definition there exists a set of $R$ column vectors $\mathbf{q}_{1}, \mathbf{q}_{2}, \ldots, \mathbf{q}_{R}$ of $\mathbf{P}$ and $R$ quad-quaternion scalars $\lambda_{1}, \lambda_{2}, \ldots, \lambda_{R}$, such that

$$
\mathbf{q}_{1} \lambda_{1}+\mathbf{q}_{2} \lambda_{2}+\cdots+\mathbf{q}_{R} \lambda_{R}=\mathbf{o}_{M \times 1} \Longleftrightarrow \lambda_{1}=\lambda_{2}=\cdots=\lambda_{R}=0 .
$$

Moreover, for an arbitrary nonzero column vector $\mathbf{q}_{R+1}$ of $\mathbf{P}$, there exist $R+1$ quad-quaternion scalars $\mu_{1}, \mu_{2}, \ldots, \mu_{R}, \mu_{R+1}$ not all zero, such that

$$
\mathbf{q}_{1} \mu_{1}+\mathbf{q}_{2} \mu_{2}+\cdots+\mathbf{q}_{R} \mu_{R}+\mathbf{q}_{R+1} \mu_{R+1}=\mathbf{o}_{M \times 1} .
$$


According to Lemma 1 and (A.1) and (A.2), we have

$$
\begin{gathered}
\chi_{q_{1}} \chi_{\lambda_{1}}+\chi_{q_{2}} \chi_{\lambda_{2}}+\cdots+\chi_{q_{R}} \chi_{\lambda_{R}} \\
=\mathbf{O}_{2 M \times 2} \Leftrightarrow \lambda_{1}=\lambda_{2}=\cdots=\lambda_{R}=0, \\
\chi_{q_{1}} \chi_{\mu_{1}}+\chi_{q_{2}} \chi_{\mu_{2}}+\cdots+\chi_{q_{R}} \chi_{\mu_{R}}+\chi_{q_{R+1}} \chi_{\mu_{R+1}}=\mathbf{O}_{2 M \times 2},
\end{gathered}
$$

where $\boldsymbol{\chi}_{q_{n}} \in\left(\mathbb{M}_{\mathbb{C}^{(J)}}\right)^{2 M \times 2}$ and $\boldsymbol{\chi}_{\lambda_{n}}, \boldsymbol{\chi}_{\mu_{n}} \in\left(\mathbb{H}_{\mathbb{C}^{(J)}}\right)^{2 \times 2}$ are the adjoint matrices of $\mathbf{q}_{n}, \lambda_{n}$ and $\mu_{n}$, respectively, $n=$ $1,2, \ldots, R, R+1$. We further denote $\boldsymbol{\chi}_{q_{n}}=\left[\boldsymbol{\chi}_{n, 0}, \boldsymbol{\chi}_{n, 1}\right], \lambda_{n}=$ $\lambda_{n, 0}+I \lambda_{n, 1}$, and $\mu_{n}=\mu_{n, 0}+I \mu_{n, 1}$, then $\chi_{1,0}, \chi_{1,1}, \ldots, \chi_{R, 0}, \chi_{R, 1}$ are column vectors of $\chi_{P}$. According to the definition of adjoint matrices and (A.3), we have

$$
\begin{gathered}
\sum_{n=1}^{R}\left(\chi_{n, 0} \lambda_{n, 0}-\chi_{n, 1} \lambda_{n, 1}\right) \\
=\mathbf{o}_{2 M \times 1} \Longleftrightarrow \lambda_{1,0}=\lambda_{1,1}=\cdots=\lambda_{R, 0}=\lambda_{R, 1}=0 \\
\sum_{n=1}^{R+1}\left(\chi_{n, 0} \mu_{n, 0}-\chi_{n, 1} \mu_{n, 1}\right)=\mathbf{o}_{2 M \times 1} \\
\sum_{n=1}^{R+1}\left(\chi_{n, 0} \mu_{n, 1}^{*}+\chi_{n, 1} \mu_{n, 0}^{*}\right)=\mathbf{o}_{2 M \times 1} .
\end{gathered}
$$

Since $\mu_{1}, \mu_{2}, \ldots, \mu_{R}, \mu_{R+1}$ are not all zero, without loss of generality, we assume $\mu_{R+1}=\mu_{R+1,0}+i \mu_{R+1,1} \neq 0$, and further assume $\mu_{R+1,1} \neq 0$. Then, we have from (A.5) that

$$
\begin{aligned}
\chi_{R+1,0} & \left(\mu_{R+1,1}^{-1} \mu_{R+1,0}^{*}+\mu_{R+1,1}^{*}\right) \\
+\sum_{n=1}^{R} & {\left[\chi_{n, 0}\left(\mu_{n, 0} \mu_{R+1,1}^{-1} \mu_{R+1,0}^{*}+\mu_{n, 1}^{*}\right)\right.} \\
& \left.\quad-\chi_{n, 1}\left(\mu_{n, 1} \mu_{R+1,1}^{-1} \mu_{R+1,0}^{*}-\mu_{n, 0}^{*}\right)\right]=\mathbf{o}_{2 M \times 1} .
\end{aligned}
$$

From (A.4) and (A.6), we see that $\chi_{1,0}, \chi_{1,1}, \ldots, \chi_{R, 0}, \chi_{R, 1}$ consist a maximal right linearly independent set, therefore the right rank of $\chi_{P}$ is $2 R$.

Proof of Lemma 7. According to Lemma 6, $\operatorname{rank}(\mathbf{Q})=$ $1 / 2 \operatorname{rank}\left(\chi_{Q}\right)$, where $\mathbf{Q}$ is a Hermitian quad-quaternion matrix that can be eigenvalue decomposed as $\mathbf{Q}=\mathbf{U D U}^{H}$. $\chi_{Q}$ is the adjoint biquaternion matrix of $\mathbf{Q}$. According to Lemma 2, $\chi_{Q}$ is also Hermitian. Then according to [37, Definition 6], we have

$$
\operatorname{rank}(\mathbf{Q})=\frac{1}{2} \operatorname{rank}\left(\chi_{Q}\right)=\frac{1}{4} \operatorname{rank}(\mathbf{D}) .
$$

Proof of Lemma 8. is directly obtained from Definition 15.

\section{ACKNOWLEDGMENT}

This work was supported in part by the National Natural Science Foundation of China under Contracts no. 60672084, no. 60602037 , and no. 60736006 .

\section{REFERENCES}

[1] R. T. Compton Jr., "The tripole antenna: an adaptive array with full polarization flexibility," IEEE Transactions on Antennas and Propagation, vol. 29, no. 6, pp. 944-952, 1981.

[2] J. Li, "Direction and polarization estimation using arrays with small loops and short dipoles," IEEE Transactions on Antennas and Propagation, vol. 41, no. 3, pp. 379-387, 1993.

[3] A. Nehorai and E. Paldi, "Vector-sensor array processing for electromagnetic source localization," IEEE Transactions on Signal Processing, vol. 42, no. 2, pp. 376-398, 1994.

[4] K. T. Wong, "Direction finding/polarization estimation-dipole and/or loop triad(s)," IEEE Transactions on Aerospace and Electronic Systems, vol. 37, no. 2, pp. 679-684, 2001.

[5] J. Tabrikian, R. Shavit, and D. Rahamim, "An efficient vector sensor configuration for source localization," IEEE Signal Processing Letters, vol. 11, no. 8, pp. 690-693, 2004.

[6] I. Ziskind and M. Wax, "Maximum likelihood localization of diversely polarized sources by simulated annealing," IEEE Transactions on Antennas and Propagation, vol. 38, no. 7, pp. 1111-1114, 1990.

[7] A. J. Weiss and B. Friedlander, "Maximum likelihood signal estimation for polarization sensitive arrays," IEEE Transactions on Antennas and Propagation, vol. 41, no. 7, pp. 918-925, 1993.

[8] J. Li and P. Stoica, "Efficient parameter estimation of partially polarized electromagnetic waves," IEEE Transactions on Signal Processing, vol. 42, no. 11, pp. 3114-3125, 1994.

[9] H. Lee and R. Stovall, "Maximum likelihood methods for determining the direction of arrival for a single electromagnetic source with unknown polarization," IEEE Transactions on Signal Processing, vol. 42, no. 2, pp. 474-479, 1994.

[10] R. O. Schmidt, A signal subspace approach to emitter location and spectral estimation, Ph.D. dissertation, Stanford University, Stanford, Calif, USA, 1981.

[11] E. R. Ferrara Jr. and T. M. Parks, "Direction finding with an array of antennas having diverse polarizations," IEEE Transactions on Antennas and Propagation, vol. 31, no. 2, pp. 231-236, 1983.

[12] Y. Hua, "A pencil-MUSIC algorithm for finding twodimensional angles and polarizations using crossed dipoles," IEEE Transactions on Antennas and Propagation, vol. 41, no. 3, pp. 370-376, 1993.

[13] A. J. Weiss and B. Friedlander, "Direction finding for diversely polarized signals using polynomial rooting," IEEE Transactions on Signal Processing, vol. 41, no. 5, pp. 1893-1905, 1993.

[14] K. T. Wong and M. D. Zoltowski, "Self-initiating MUSICbased direction finding and polarization estimation in spatiopolarizational beamspace," IEEE Transactions on Antennas and Propagation, vol. 48, no. 8, pp. 1235-1245, 2000.

[15] K. T. Wong, L. Li, and M. D. Zoltowski, "Root-MUSIC-based direction-finding and polarization estimation using diversely polarized possibly collocated antennas," IEEE Antennas and Wireless Propagation Letters, vol. 3, no. 1, pp. 129-132, 2004.

[16] M. D. Zoltowski and K. T. Wong, "Closed-form eigenstructure-based direction finding using arbitrary but identical subarrays on a sparse uniform Cartesian array grid," IEEE Transactions on Signal Processing, vol. 48, no. 8, pp. 2205-2210, 2000.

[17] A. Swindlehurst and M. Viberg, "Subspace fitting with diversely polarized antenna arrays," IEEE Transactions on Antennas and Propagation, vol. 41, no. 12, pp. 1687-1694, 1993. 
[18] J. Li, P. Stoica, and D. Zheng, "Efficient direction and polarization estimation with a COLD array," IEEE Transactions on Antennas and Propagation, vol. 44, no. 4, pp. 539-547, 1996.

[19] R. Roy and T. Kailath, "ESPRIT-estimation of signal parameters via rotational invariance techniques," IEEE Transactions on Acoustics, Speech and Signal Processing, vol. 37, no. 7, pp. 984-995, 1989.

[20] J. Li and R. T. Compton Jr., "Angle and polarization estimation using ESPRIT with a polarization sensitive array," IEEE Transactions on Antennas and Propagation, vol. 39, no. 9, pp. 1376-1383, 1991.

[21] J. Li and R. T. Compton Jr., "Angle estimation using a polarization sensitive array," IEEE Transactions on Antennas and Propagation, vol. 39, no. 10, pp. 1539-1543, 1991.

[22] J. Li and R. T. Compton Jr., "Two-dimensional angle and polarization estimation using the ESPRIT algorithm," IEEE Transactions on Antennas and Propagation, vol. 40, no. 5, pp. 550-555, 1992.

[23] J. Li, "On polarization estimation using a crossed-dipole array," IEEE Transactions on Signal Processing, vol. 42, no. 4, pp. 977-980, 1994.

[24] K. T. Wong and M. D. Zoltowski, "Uni-vector-sensor ESPRIT for multisource azimuth, elevation, and polarization estimation," IEEE Transactions on Antennas and Propagation, vol. 45, no. 10, pp. 1467-1474, 1997.

[25] K. T. Wong and M. D. Zoltowski, "Closed-form direction finding and polarization estimation with arbitrarily spaced electromagnetic vector-sensors at unknown locations," IEEE Transactions on Antennas and Propagation, vol. 48, no. 5, pp. 671-681, 2000.

[26] M. D. Zoltowski and K. T. Wong, "ESPRIT-based 2-D direction finding with a sparse uniform array of electromagnetic vector sensors," IEEE Transactions on Signal Processing, vol. 48, no. 8, pp. 2195-2204, 2000.

[27] K.-C. Tan, K.-C. Ho, and A. Nehorai, "Uniqueness study of measurements obtainable with arrays of electromagnetic vector sensors," IEEE Transactions on Signal Processing, vol. 44, no. 4, pp. 1036-1039, 1996.

[28] K.-C. Tan, K.-C. Ho, and A. Nehorai, "Linear independence of steering vectors of an electromagnetic vector sensor," IEEE Transactions on Signal Processing, vol. 44, no. 12, pp. 30993107, 1996.

[29] B. Hochwald and A. Nehorai, "Identifiability in array processing models with vector-sensor applications," IEEE Transactions on Signal Processing, vol. 44, no. 1, pp. 83-95, 1996.

[30] D. Rahamim, J. Tabrikian, and R. Shavit, "Source localization using vector sensor array in a multipath environment," IEEE Transactions on Signal Processing, vol. 52, no. 11, pp. 30963103, 2004

[31] Y. Zhang, B. A. Obeidat, and M. G. Amin, "Spatial polarimetric time-frequency distributions for direction-of-arrival estimations," IEEE Transactions on Signal Processing, vol. 54, no. 4, pp. 1327-1340, 2006.

[32] Y. Xu and Z. Liu, "Polarimetric angular smoothing algorithm for an electromagnetic vector-sensor array," IET Radar, Sonar \& Navigation, vol. 1, no. 3, pp. 230-240, 2007.

[33] P. Chevalier, A. Ferréol, L. Albera, and G. Birot, "Higher order direction finding from arrays with diversely polarized antennas: the PD-2q-MUSIC algorithms," IEEE Transactions on Signal Processing, vol. 55, no. 11, pp. 5337-5350, 2007.

[34] Y. Wu, H. C. So, C. Hou, and J. Li, "Passive localization of near-field sources with a polarization sensitive array," IEEE Transactions on Antennas and Propagation, vol. 55, no. 8, pp. 2402-2408, 2007.
[35] N. Le Bihan and J. I. Mars, "Singular value decomposition of quaternion matrices: a new tool for vector-sensor signal processing," Signal Processing, vol. 84, no. 7, pp. 1177-1199, 2004.

[36] S. Miron, N. Le Bihan, and J. I. Mars, "Quaternion-MUSIC for vector-sensor array processing," IEEE Transactions on Signal Processing, vol. 54, no. 4, pp. 1218-1229, 2006.

[37] N. Le Bihan, S. Miron, and J. I. Mars, "MUSIC algorithm for vector-sensors array using biquaternions," IEEE Transactions on Signal Processing, vol. 55, no. 9, pp. 4523-4533, 2007.

[38] J. Ward, Quaternions and Cayley Numbers: Algebra and Applications, Kluwer Academic Publishers, New York, NY, USA, 1997.

[39] K. Imaeda and M. Imaeda, "Sedenions: algebra and analysis," Applied Mathematics and Computation, vol. 115, no. 2-3, pp. 77-88, 2000.

[40] T. Y. Lam, D. B. Leep, and J.-P. Tignol, "Biquaternion algebras and quartic extensions," Publications Mathématiques de l'Institut des Hautes Études Scientifiques, vol. 77, no. 1, pp. 63-102, 1993.

[41] A. A. Albert, "A construction of non-cyclic normal division algebras," Bulletin of the American Mathematical Society, vol. 38, no. 6, pp. 449-456, 1932.

[42] T. Unger and N. Markin, "Quadratic forms and space-time block codes from generalized quaternion and biquaternion algebras," http://arxiv.org/abs/0807.0199.

[43] W. R. Hamilton, "On a new species of imaginary quantities connected with a theory of quaternions," Proceedings of the Royal Irish Academy, vol. 2, pp. 424-434, 1843. 